\title{
Matching as Nonparametric Preprocessing for Reducing Model Dependence in Parametric Causal Inference
}

\author{
Daniel E. Ho \\ Stanford Law School, 559 Nathan Abbott Way, Stanford, CA 94305 \\ e-mail: dho@law.stanford.edu \\ Kosuke Imai \\ Department of Politics, Princeton University, \\ Princeton, NJ 08544 \\ e-mail: kimai@princeton.edu \\ Gary King \\ Department of Government, Harvard University, \\ 1737 Cambridge Street, Cambridge, MA 02138 \\ e-mail: king@harvard.edu (corresponding author) \\ Elizabeth A. Stuart \\ Departments of Mental Health and Biostatistics, \\ Johns Hopkins Bloomberg School of Public Health, \\ 624 North Broadway, Room 804, Baltimore, MD 21205 \\ e-mail: estuart@jhsph.edu
}

\begin{abstract}
Although published works rarely include causal estimates from more than a few model specifications, authors usually choose the presented estimates from numerous trial runs readers never see. Given the often large variation in estimates across choices of control variables, functional forms, and other modeling assumptions, how can researchers ensure that the few estimates presented are accurate or representative? How do readers know that publications are not merely demonstrations that it is possible to find a specification that fits the author's favorite hypothesis? And how do we evaluate or even define statistical properties like unbiasedness or mean squared error when no unique model or estimator even exists? Matching methods, which offer the promise of causal inference with fewer assumptions, constitute one possible way forward, but crucial results in this fast-growing methodological literature are often grossly misinterpreted. We explain how to avoid these
\end{abstract}

Authors' note: Our thanks to Dan Carpenter and Jeff Koch for data; Alberto Abadie, Neal Beck, Sam Cook, Alexis Diamond, Ben Hansen, Guido Imbens, Olivia Lau, Gabe Lenz, Paul Rosenbaum, Don Rubin, and Jas Sekhon for many helpful comments; and the National Institutes of Aging (P01 AG17625-01), the National Institute of Mental Health (MH066247), the National Science Foundation (SES-0318275, IIS-9874747, SES0550873), and the Princeton University Committee on Research in the Humanities and Social Sciences for research support. Software to implement the methods in this paper is available at http://GKing.Harvard.edu/ matchit and a replication data file is available as Ho et al. (2006).

(c) The Author 2007. Published by Oxford University Press on behalf of the Society for Political Methodology.

All rights reserved. For Permissions, please email: journals.permissions@oxfordjournals.org 
misinterpretations and propose a unified approach that makes it possible for researchers to preprocess data with matching (such as with the easy-to-use software we offer) and then to apply the best parametric techniques they would have used anyway. This procedure makes parametric models produce more accurate and considerably less model-dependent causal inferences.

\section{Introduction}

Political science research typically begins by first spending considerable time collecting, correcting, recollecting, merging, and recoding data. When all the data are finally available in the right format and loaded into one's favorite statistical package, researchers obtain a causal estimate by running some parametric statistical procedure-linear regression, logit, probit, duration models, structural equation models, count models, etc. This run typically takes only a few seconds and, according to some textbooks, it would be time to write up the results. Of course, this never happens. Instead, we do a second run with different control variables, a third with a different functional form, a fourth with a different measure of our key causal variable, one with different sample periods or observation subsets, and then each of these and others are repeated with slight variations over and over again. Although this usual procedure produces hundreds or thousands of alternative estimates of our single causal effect, we typically only choose one, and rarely more than $5-10$, to present in a paper. Yet, we know that our estimates depend on their corresponding modeling assumptions and that different specifications can yield very different causal inferences.

Most causal effect estimates given in the literature are thus model dependent, at least to some degree, but at the same time the statistical properties of most estimators used by political scientists depend on the assumption that we know the single correct model. Model dependence combined with likelihood, Bayesian, or other methods that condition on a single model, framework, or specification mean that we cannot logically even ask whether an estimator has desirable properties, such as unbiasedness, consistency, efficiency, mean squared error, etc., since a unique estimator must exist before it can be evaluated. The related practical problem for researchers is how to convince readers that we picked the right specification rather than the one that most supported our favorite hypothesis. What does it even mean to choose "the right" parametric model estimates when any chosen model depends on assumptions we cannot verify? When we read a published article, how do we know whether the causal effect presented is accurate or whether the article merely constitutes a demonstration that it is possible to find a specification consistent with the author's prior expectations? King and Zeng (2006) offer methods to detect the problem of model dependence; we show how to ameliorate it, when possible.

We begin where virtually all causal inference methods based on observational data begin, by making the ignorability (or no omitted variable bias) assumption and conditioning on the definition of a key causal (or "treatment") variable and a set of variables we choose to control for. From this common starting point, we offer an easy method of adjusting for as much of the information in these control variables as possible without parametric assumptions. We do this by preprocessing a data set with matching methods so that the treated group is as similar as possible to the control group. In the preprocessed data set, the treatment variable is closer to being independent of the background covariates, which renders any subsequent parametric adjustment either irrelevant or less important.

This inferential strategy has three key advantages. First, the approach is easy to use since researchers need not give up their familiar parametric analysis methods. Instead, they 
merely add a simple preprocessing step before the parametric analysis procedures they would have used anyway (or should have used anyway if they are not already following best practices). All of the intuition, diagnostics, uncertainty estimates, and knowledge about parametric procedures can then be used as before. Second, by breaking or reducing the link between the treatment variable and control variables, preprocessing makes estimates based on the subsequent parametric analyses far less dependent on modeling choices and specifications. When the data are of sufficiently high quality so that proper matches are available (in a manner we define below), causal effect estimates do not vary much even when changing parametric modeling assumptions. Finally, since most of the adjustment for potentially confounding control variables is done nonparametrically, the potential for bias is greatly reduced compared to parametric analyses based on raw data. Furthermore, in many situations, the same preprocessing also leads to a reduction in the variance of the estimated causal effects, and so the mean squared error will normally be lower too.

In a sense, our recommendations already constitute current best practice since matching alone is not a method of estimation and always requires some technique after matching to compute estimates. The problem is that the method most commonly chosen after matching has been a simple difference in means without any controls for potential confounding variables. We simply point out that, except in the extraordinary case where matching is exact, common parametric procedures have the potential to greatly improve causal inferences even after matching.

The fast-growing matching literature is theoretically sophisticated, but, from the point of view of the practical researcher, it looks like a cacophony of conflicting techniques, practices, conventions, and rules of thumb. Confusion reigns over computing standard errors and confidence intervals due to apparently contradictory differences stemming from alternative nonparametric and parametric perspectives. Many theoretical results do not apply to practical applications unless unknown theoretical quantities or specifications are somehow divined. Perhaps as a consequence, some of the most important theoretical results are routinely misinterpreted in applied research and even in some theoretical statistical work. Coherent guidelines for practice are often conflicting, absent, or misunderstood. We try to clarify the key misunderstandings in this literature and to present a coherent unified perspective for applied research. ${ }^{1}$

Our approach is similar in spirit to, or a generalization of the ideas in, Cochran and Rubin (1973), Rubin (1973, 1979), Rosenbaum and Rubin (1984), Rubin and Thomas (2000), and Imai and van Dyk (2004) who each recommend matching followed by a different form of parametric adjustment, as well as strategies used in applied research problems by Rosenbaum (1986) and others, as discussed in Glazerman, Levy, and Myers (2003). ${ }^{2}$ To our knowledge, the present paper is the first to propose and work out the

\footnotetext{
${ }^{1}$ Although matching methods now comprise a substantial fraction of the empirical work in observational studies in some disciplines, such as epidemiology and medicine, the diversity of substantive applications and the conflicting methodological languages used to describe the same underlying concepts have limited the spread of these powerful techniques to much of the social sciences. However, the misunderstandings we discuss here are no less prevalent in these other areas.

${ }^{2}$ It is also similar to Heckman, Ichimura, and Todd (1998) who developed forms of matching combined with semiparametric (kernel weighting) analyses, as well as to the parametric bias adjustment for one form of matching by Abadie and Imbens (2006b), except that, to avoid inducing new biases, we recommend below that matching be evaluated prior to examining the dependent variable, which is not the case with these latter approaches as generally implemented. Our idea is also similar in spirit to methods in other areas that preprocess data so that subsequent analyses can be improved without modifying existing techniques, such as multiple imputation (Rubin 1987; King et al. 2001) and outlier and feature detection (Bishop 1995, chap. 8).
} 
conditions for matching as a general method of nonparametric preprocessing, suitable for improving any parametric method.

Our general preprocessing strategy also made it possible for us to write easy-to-use software that implements all the ideas discussed in this paper and incorporates most existing approaches described in the literature. The program, called MatchIt, is available as an open source and free R package at http://gking.harvard.edu/matchit (see Appendix); MatchIt also works seamlessly with the general purpose $\mathrm{R}$ statistics package called Zelig (Imai, King, and Lau 2006).

Other approaches to reducing model dependence include weighting (Hirano, Imbens, and Ridder 2003; Robins and Rotnitzky forthcoming), nonparametric techniques, robust estimation, and fitting checks for parametric models. Although when used properly each of these approaches can reduce model dependence, matching may be simpler to use and understand and would work as we suggest here to improve all the parametric models now used for making causal inferences in the social sciences. More importantly, since these alternative approaches also involve specification or modeling decisions, preprocessing via matching works well in combination with these approaches too, and so they should not be considered competitors. Other seemingly possible alternatives, such as Bayesian model averaging (Hoeting et al. 1999; Imai and King 2004) and cross-validation (Black and Smith 2004), are useful for predictive inference but not directly applicable in the context of causal inference.

\section{Definition of Causal Effects}

The notation, ideas, and running example in this section parallel that in King, Keohane, and Verba (1994, sec. 3.1.1), but key aspects of the ideas originate with many others, especially Neyman (1923), Fisher (1935), Cox (1958), Rubin (1974), and Holland (1986) in statistics; Roy (1951) and Quandt (1972) in econometrics; and Lewis (1973) in philosophy. The most important idea in this section is that a causal effect is a theoretical quantity, defined independently of any empirical method that might be used to estimate it from real data.

Our running example in this section is estimating the electoral advantage of incumbency for Democrats in the U.S. House of Representatives. In most of the methodological literature on causal inference, researchers simplify the exposition by considering only a single dichotomous causal (or treatment) variable. We do the same and label it $T_{i}$, which takes a value of 1 if congressional district $i(i=1, \ldots, n)$ receives the treatment and 0 if $i$ is untreated (the "control condition"). In our example, the treatment is whether the Democratic incumbent receives the party's nomination in district $i$.

The observed outcome (or "dependent") variable is $y_{i}$, which in our case is the Democratic proportion of the two-party vote in district $i$. Finally, each district $i$ has a variety of characteristics determined prior to the incumbent's decision to run for election and the party's decision to renominate the incumbent, some of which we measure and collect in a vector denoted $X_{i}$. Whether preprocessing or not, variables that are even in part a consequence of the treatment variable should never be controlled for when estimating a causal effect (see Cox 1958, sec. 4.2; Rosenbaum 1984; Rosenbaum 2002, 73-4). This is of course a critical point since controlling for the consequences of a causal variable can severely bias a causal inference. For example, controlling for aggregate voting intentions the day before the election would obviously control away, and thus bias, the estimated incumbency effect. This "posttreatment bias" problem is far too common in many areas of political science (King and Zeng 2007). 
To clarify our inferential goals, we begin by defining the "fixed causal effect," which is the simplest in-sample definition available, in that it is the closest to the data. We then generalize the definition to include features of random causal effects that are useful for understanding connections between nonparametric preprocessing and parametric models, define causal effects of interest at the population level, and discuss multiple and nonbinary treatments.

\subsection{Fixed Causal Effects}

Because of pretreatment differences among the districts (both measured, $X_{i}$, and unmeasured), the causal effect may also differ across the districts. We therefore define the casual effect at the district (i.e., observation) level.

A causal effect is a function of potential outcomes: let $y_{i}(1) \equiv y_{i}\left(T_{i}=1\right)$ be the vote we would observe in district $i$ in say the 2008 election if in fact the Democratic incumbent receives his or her party's nomination (i.e., $\left.T_{i}=1\right)$, and let $y_{i}(0) \equiv y_{i}\left(T_{i}=0\right)$ be the vote we would observe if the Democratic Party nominates a nonincumbent (i.e., $T_{i}=0$ ). (Each of the potential vote outcomes in district $i$ is thus a function of the incumbency status in the same district, $T_{i}$, and not a function of candidates in other districts.) The use of parentheses in this notation denotes that the outcome is potential, and so not necessarily observed, and that it depends on the value of the variable in parentheses. Since these are potential outcomes, their values remain the same regardless of whether the treatment is in fact applied in district $i$ or not.

The difference between the two potential outcomes defines the fixed (or unit specific) causal effect

$$
\text { Fixed causal effect for unit } i \equiv y_{i}(1)-y_{i}(0) \text {. }
$$

(This quantity is an unobserved realization of a random variable to be defined below.) Since the Democratic Party will either nominate $\left(T_{i}=1\right)$ or not nominate $\left(T_{i}=0\right)$ an incumbent to run in district $i$, one of these potential outcomes is always a counterfactual and thus never observed. This is known as the "fundamental problem of causal inference" (Holland 1986).

\subsection{Random Causal Effects}

Now, imagine that the potential outcomes in equation (1) are realizations of corresponding random variables (for which we use the corresponding capital letters). This produces the random causal effect

$$
\text { Random causal effect for unit } i \equiv Y_{i}(1)-Y_{i}(0),
$$

features of which constitute alternative quantities of interest. For example, our second causal effect is the mean causal effect, which is the average over repeated hypothetical draws of the random causal effect

$$
\begin{aligned}
\text { Mean causal effect } & \equiv E(\text { random causal effect }) \\
& =E\left[Y_{i}(1)-Y_{i}(0)\right] \\
& =\mu_{1}-\mu_{0}
\end{aligned}
$$

where $\mu_{1} \equiv E\left[Y_{i}(1)\right]$ and $\mu_{0} \equiv E\left[Y_{i}(0)\right]$. 


\subsection{Average Quantities of Interest}

In most applications, estimating the treatment effect for each observation is unnecessary; instead, the goal is to estimate the average effect over all observations, for some subset of observations or for a particular population. This leads to several choices for quantities of interest, each of which is defined for either fixed (in-sample) or population causal effects. We focus on in-sample effects-that is, based on quantities of interest for all or a subset of units in our data-since they are closer to the data than effects for averages of specified populations or population subgroups. However, even for survey researchers and others interested in population quantities, the practical difference between population and insample estimators is not normally important since a good estimator for one is "automatically a good estimator for the other" (Imbens 2004, 6).

Among in-sample effects, we consider two choices. The first is the average treatment effect or ATE

$$
\begin{aligned}
\mathrm{ATE} & \equiv \frac{1}{n} \sum_{i=1}^{n} E\left[Y_{i}(1)-Y_{i}(0) \mid X_{i}\right] \\
& =\frac{1}{n} \sum_{i=1}^{n} \mu_{1}\left(X_{i}\right)-\mu_{0}\left(X_{i}\right),
\end{aligned}
$$

where $\mu_{0}\left(X_{i}\right) \equiv E\left[Y_{i}(0) \mid X_{i}\right]$ and $\mu_{1}\left(X_{i}\right) \equiv E\left[Y_{i}(1) \mid X_{i}\right]$. This quantity is the mean causal effect for a unit whose characteristics are represented by $X_{i}$, averaged over all units (so that the expectation operator in the first-line averages over the random causal effects for each unit with the values of the pretreatment variable equal to $X_{i}$, and the summation over $i$ in both lines refers to the observed sample).

Often, of more interest substantively is the average treatment effect on the treated or ATT

$$
\begin{aligned}
\mathrm{ATT} & \equiv \frac{1}{\sum_{i=1}^{n} T_{i}} \sum_{i=1}^{n} T_{i} E\left[Y_{i}(1)-Y_{i}(0) \mid X_{i}\right] \\
& =\frac{1}{\sum_{i=1}^{n} T_{i}} \sum_{i=1}^{n} T_{i}\left[\mu_{1}\left(X_{i}\right)-\mu_{0}\left(X_{i}\right)\right] .
\end{aligned}
$$

In our example, this is the average causal effect in districts in which the Democratic Party nominated the incumbent member of the House. From one perspective, we might want to know this treatment effect on the treated (the ATT) since obviously this is the group of districts where the treatment was applied. In other words, the ATT is the effect of the treatment actually applied. Medical studies typically use the ATT as the designated quantity of interest because they often only care about the causal effect of drugs for patients that receive or would receive the drugs. For another example, in job training programs, we are not normally interested in assigning employed people to have this training (Heckman, Ichimura, and Todd 1998). In the social sciences, the ATE is often a reasonable choice, as is the ATT. In our example, we might be interested not only in the effect of incumbency when the incumbent is nominated, but we can also imagine what might have happened if an incumbent were nominated in a district in which he or she did not actually receive the nomination. In this paper, we usually focus on ATT as the quantity of interest when it is conceptually or algebraically simpler, but we also show how to compute the ATE. If causal effects are constant over $i$, then the ATT and the ATE are identical. 
The ATE and ATT defined above are each in-sample, unconditional estimates. Alternative quantities of interest are defined by making the choice of unconditional versus conditional and in-sample versus population quantities. In contrast to the unconditional quantities above, conditional ATE and ATT quantities get even closer to the data by conditioning each on $y_{i}$. The result is that $Y_{i}(1)$ and $Y_{i}(0)$ in the expressions above are replaced by $y_{i}(1)$ and $y_{i}(0)$, for each $i$. Then for each $i$, only one of the counterfactual outcomes, $y_{i}(1)$ or $y_{i}(0)$, is estimated and the other is set to the observed $y_{i}$. These conditional, in-sample ATE and ATT quantities are reasonable alternatives that we often use.

Similarly, to change from in-sample quantities in the expressions above to the population ATE, we average it over (i.e., weight by) the population distribution of $X$; for the ATT, we average over the conditional distribution of $X$ given $T=1$. Point estimates for the population and sample are normally identical, although the variances for the population estimates are usually larger.

\subsection{Nonbinary and Multiple Treatments}

Projects with a causal variable that has more than two categories or is continuous or mixed can dichotomize (perhaps in several alternative ways) or use more complicated methods designed especially for these variables (Imai and van Dyk 2004). Those with more than one causal variable of interest can follow all the advice herein for one variable at a time, which would involve matching separately for each and working hard to avoid posttreatment bias in the process. We stick to a single binary treatment here since it greatly simplifies the exposition and improves intuition even for those who will ultimately use more sophisticated treatments.

\section{Assumptions and Data Collection Mechanisms}

We now describe the assumptions necessary for making causal inferences in experimental and observational research. Some version of these assumptions, or some way to deal with the information in them, is necessary no matter what statistical methods are used for estimation. Any specific statistical method chosen will make additional assumptions, but the ones discussed here affect essentially all methods.

\subsection{Experimental Research}

Although classical randomized experiments are only rarely conducted in political science, they remain a useful ideal type for understanding other research designs. Indeed, the preprocessing procedures we recommend alter the data to make them more like what we would have seen if an experiment had been conducted.

Valid and relatively straightforward causal inferences can be achieved via classical randomized experiments. Such experiments have three critical features: (1) random selection of units to be observed from a given population, (2) random assignment of values of the treatment to each observed unit, ${ }^{3}$ and (3) large $n$ (sample size).

Feature (1) avoids selection bias by identifying a given population and guaranteeing that the probability of selection from this population is related to the potential outcomes only by random chance. Combining Feature (1) with the large $n$ from Feature (3) guarantees that the chance that something will go wrong is vanishingly small.

\footnotetext{
${ }^{3}$ Below, we assume a classical randomized experiment with simple random assignment rather than random assignment based on some pretreatment covariates. If treatment assignment depends on observed values of the covariates, those covariates should be taken into account in subsequent analyses.
} 
Random assignment in Feature (2) guarantees the absence of omitted variable bias even without any control variables included. To see this, recall that under the usual econometric conditions for omitted variable bias, a variable $X_{i}$ must be controlled for if it is causally prior to $T_{i}$, empirically related to $T_{i}$, and affects $Y_{i}$ conditional on $T_{i}$. If instead one or more of the three conditions do not hold, then $X_{i}$ may be omitted without any resulting bias (although the variance may increase). Random assignment guarantees that $T_{i}$ is independent of any $X_{i}$, whether measured or not, except by random chance. Moreover, the large $n$ in Feature (3) guarantees that this chance is vanishingly small.

Classical randomized experiments are a true ideal type, particularly in relation to most social science research, which almost always fails to meet at least one of the three features. Even most social science laboratory experiments have random assignment but no random selection and often a small $n$. Traditional survey research has what is intended to be random selection (although with dramatically increasing nonresponse rates and cell phone usage, this is a less plausible claim) and certainly has a large $n$, but random assignment, except when the treatment involves the wording of survey questions, is usually impossible.

\subsection{Observational Research}

We define observational data collection mechanisms as any process generating data that does not meet all three features of a classical randomized experiment. Scholars trying to use the experimental paradigm attempt to design research to meet all three features discussed in the previous section. Researchers analyzing observational data are instead forced to make assumptions that, if correct, help them avoid various threats to the validity of their causal inferences. ${ }^{4}$

In this paper, we assume data are selected in a manner that does not generate selection bias. Observations need not be selected at random, as in an experiment, but the probability of selection must not depend on potential outcomes. This can be satisfied by carefully considering and controlling for the sample selection process (as in case-control designs) or by changing the quantity of interest to be that reflected by the sample. However it is done, avoiding selection bias is the subject of a great deal of concern and study in a large variety of methodological and substantive literatures. We mention it here to emphasize that all the well-known concerns about selecting on the dependent variable should remain a concern to researchers even when adopting our approach of preprocessing data via nonparametric matching procedures.

We also assume that researchers analyzing observational data have sufficient information in their measured pretreatment control variables $X_{i}$ so that it is possible via some method to make valid causal inferences. This is known in political methodology and econometrics as the absence of "omitted variable bias," so that $X_{i}$ must include all variables that are causally prior to $T_{i}$, associated with $T_{i}$, and affect $Y_{i}$ conditional on $T_{i}$ (Goldberger 1991; King, Keohane, and Verba 1994), or "selection on observables" (Heckman and Robb 1985). In statistics, this same condition is known as "ignorability," which means that $T_{i}$ and the unobserved potential outcomes are independent after conditioning on $X_{i}$ and the observed potential outcomes, and so we can literally ignore all unobserved variables (Rubin 1978). In biostatistics, it is known as the absence of "unmeasured confounding," and in several fields it is known as "conditional independence." Whatever the name, it is a strong condition, but it is one about which social scientists are

\footnotetext{
${ }^{4}$ Our definition of "observational data" is more expansive than some. In some fields, only deviations from random assignment would be called observational. Our definition emphasizes the necessity of assumptions in general rather than any ones in particular.
} 
deeply knowledgeable and it is the central methodological concern of many substantive scholarly articles. We emphasize this assumption to make clear that our procedures contain no magic: They do not help us control for variables that are not measured.

In the context of the no selection or omitted variable bias assumptions, we have implicitly made three others that are worth additional emphasis here. First, the pretreatment covariates $X_{i}$ are truly pretreatment and are thus not consequences of $T_{i}$. Second, we assume the independence of units, which is the equivalent of assuming the absence of time series or spatial autocorrelation across units (or in other words that the two potential outcomes for observation $i$ and the treatment for observation $j$ are independent, for all $i \neq j$ ). We have also assumed that the treatment administered to each unit is the same. This assumption would be violated in our example if incumbency status meant something different across districts. ${ }^{5}$

Satisfying the assumptions discussed in this section still leaves many other assumptions to be made when choosing a specific statistical inference method. We now focus on this point in the context of commonly used parametric methods.

\section{Parametric Analysis Methods}

Researchers willing to assume that a particular parametric model (up to some unknown parameters) generated their data should specify and directly estimate this model. Preprocessing data with matching procedures will not help in this situation. Of course, few researchers with observational data sets have this kind of knowledge, and as a result some choices need to be made among the range of possible parametric models. The dilemma is that although researchers using parametric methods do not know the true parametric model, they must proceed as if they do.

We begin by specifying a single but general parametric model that characterizes the range of models that researchers might choose from. The special cases of this model include almost all parametric models that have been used in the social sciences. First, define the chosen stochastic component for the model as $Y_{i} \mid T_{i}, X_{i} \sim p\left(\mu_{T_{i}}\left(X_{i}\right), \theta\right)$ for probability density $p(\cdot)$, mean $\mu_{T_{i}}\left(X_{i}\right)$, and vector of ancillary parameters $\theta$. Then, denote the systematic component as $\mu_{T_{i}}\left(X_{i}\right) \equiv E\left[Y_{i} \mid T_{i}, X_{i}\right]=g\left(\alpha+T_{i} \beta+X_{i} \gamma\right)$ for some specified functional form $g(\cdot)$ and with intercept $\alpha$ and coefficients $\beta$ and $\gamma$. The ancillary parameters may also be specified to vary over observations as a function of $X_{i}$ or other covariates. This framework includes all generalized linear models (McCullagh and Nelder 1989), as well as many others. For example, if $p(\cdot)$ is normal and $g(c)=c$, we have linear regression; if $p(\cdot)$ is Bernoulli and $g(c)=1 /\left(1+\mathrm{e}^{-c}\right)$, the model reduces to a logistic regression.

We define the ATT in equation (6) under this model by substituting in the definitions of the potential outcomes from the systematic component, with $T_{i}$ taking on values 1 and 0 , respectively

$$
\begin{aligned}
& \mu_{1}\left(X_{i}\right) \equiv E\left[Y_{i}(1) \mid T_{i}=1, X_{i}\right]=g\left(\alpha+\beta+X_{i} \gamma\right), \\
& \mu_{0}\left(X_{i}\right) \equiv E\left[Y_{i}(0) \mid T_{i}=0, X_{i}\right]=g\left(\alpha+X_{i} \gamma\right) .
\end{aligned}
$$

We can produce estimates of these quantities by assuming independence over observations and forming a likelihood function

\footnotetext{
${ }^{5}$ These last two assumptions are sometimes known as the "stable unit treatment value assumption" or SUTVA (Rubin 1974).
} 


$$
L(\alpha, \beta, \gamma, \theta \mid Y, T, X)=\prod_{i=1}^{n} p\left(Y_{i} \mid g\left(\alpha+T_{i} \beta+X_{i} \gamma\right), \theta\right)
$$

the maximum of which gives parameter estimates, or via Bayesian or other inferential methods.

We now turn to the difficulties in making causal inferences from experimental versus observational data under this general model and conclude this section with an illustration and formal definition of model dependence.

\subsection{Experimental Data}

In experimental data, random assignment guarantees (among other things) that $T_{i}$ and (any observed or unobserved) $X_{i}$ are independent. In this situation, $X_{i}$ cannot be a confounding factor when estimating the effect of $T_{i}$, and we drop $X_{i}$ and replace equation (7) with

$$
\begin{aligned}
& E\left[Y_{i}(1) \mid T_{i}=1\right] \equiv \mu_{1}=g(\alpha+\beta) \\
& E\left[Y_{i}(0) \mid T_{i}=0\right] \equiv \mu_{0}=g(\alpha)
\end{aligned}
$$

and the ATT in equation (6) with

$$
\mathrm{ATT}=g(\alpha+\beta)-g(\alpha)
$$

which importantly no longer has a summation sign over $i$.

The systematic components in equation (9) are now scalar constants for all $i$. This is a key result since the functional form $g(\cdot)$ no longer models a high-dimensional space representing how the mean varies over $i$ as a function of all the variables in $X_{i}$ but instead now merely amounts to a simple scalar reparameterization. The fact that $g(\cdot)$ is now a scalar is central: Since maximum likelihood is invariant to reparameterization-meaning, for example, that the maximum likelihood estimate (MLE) of $\alpha$ is the same as the positive square root of the MLE of $\alpha^{2}$ (King 1989, 75-6) - we get the same estimate of the expected potential outcomes no matter how $g(\cdot)$ is defined. ${ }^{6}$ When $\mu_{0}$ and $\mu_{1}$ are a function of $X_{i}$, the choice of $g(\cdot)$ is a difficult substantive decision typically requiring more knowledge than is available. In contrast, in experimental data, because we can now drop $X_{i}$, the choice of $g(\cdot)$ reduces to an easy computational issue with no substantive import. Moreover, given any chosen stochastic component, this result holds for a wide range of parametric models, including all the special cases of the general model given above.

Since the specific maximum likelihood estimator of the population mean for many common probability densities is merely the sample mean, the analysis of classical randomized experiments typically comes down to taking the difference in the sample means of $Y_{i}$ for the treatment and control groups. But even if one chooses to run a parametric model (for reasons of efficiency, conducting conditional inferences, or because of knowledge of the functional form), the absence of model dependence means that the choice for a functional form will not matter: The results will be almost the same no matter what choice one makes for the functional form.

\footnotetext{
${ }^{6}$ To rule out degenerate cases such as $g(a)=8$, we require that the image of $g(\cdot)$ and the range of the potential outcomes be the same.
} 


\subsection{Observational Data}

In experiments, random assignment breaks the link between $T_{i}$ and $X_{i}$, eliminating the problem of model dependence. When analyzing observational data with parametric methods, we are not so fortunate. We cannot reduce equation (7) to equation (9) and so are left having to model the full functional relationship that connects the mean as it varies as a function of $T_{i}$ and $X_{i}$ over observations. Since $X_{i}$ is typically multidimensional, this is a surprisingly difficult task with rather severe consequences for research practice.

The problem is the curse of dimensionality and the consequence in practice is model dependence. We begin with the former and for simplicity suppose that we have a continuous dependent variable and one 10-category explanatory variable, and our goal is to use linear regression to estimate the functional relationship without actually making functional form assumptions. To do this, we represent the 10 categories with 10 parameters (a constant and nine dummy variables or equivalently 10 mean indicator variables). In contrast, the usual approach to estimation is to assume linearity by directly including the 10-category variable. This enables us to enter not 10 indicator variables, but rather only a constant term and one slope coefficient. How do we get from 10 parameters to only two? Pure assumption. If we have some sense that the relationship is indeed linear or close to linear, this is a good use of external information to reduce the number of parameters that must be estimated. If not, then we still have the best linear approximation to the conditional expectation function, but the relationship we estimate can be far off. If we are running this regression for the purpose of estimating a causal effect, then the treatment variable is also in the regression, and its coefficient can be biased to any degree if the functional relationship with the control variables is misspecified.

This problem quickly becomes more serious as the number of explanatory variables increases. For example, estimation without functional form assumptions with two 10category explanatory variables would require not 20 parameters but 100 . In this case, the usual approach would include a constant term and two slope coefficients, reducing 100 parameters to three by pure assumption. And with multiple explanatory variables, claims about external knowledge constraining the functional form much become dubious. In this example, by what theory would we know that 97 parameters, representing every form of nonlinearity and interaction, should be set to exactly zero? Including a linear interaction would not help much since it would merely add one more parameter to estimate, and so we would still need to make assumptions about the remaining 96 parameters.

Estimating rather than making assumptions about all these extra parameters is obviously not possible under the standard regression approach since social science data sets do not come with anywhere near enough observations. We cannot avoid the problem with nonlinear or nonnormal statistical models since these pose the same curse of dimensionality as linear regression. The assumption of ignorability, which enables us to make the positivist assumption that we have measured and observed all necessary variables, is insufficient.

Instead, we are led to the inescapable conclusion that, in parametric causal inference of observational data, many assumptions about many parameters are frequently necessary, and only rarely do we have sufficient external information to make these assumptions based on genuine knowledge. The frequent, unavoidable consequence is high levels of model dependence, with no good reason to choose one set of assumptions over another. Residual and other diagnostics will uncover some forms of misspecification, but the curse of dimensionality prevents any simple parametric solution to the problem. 

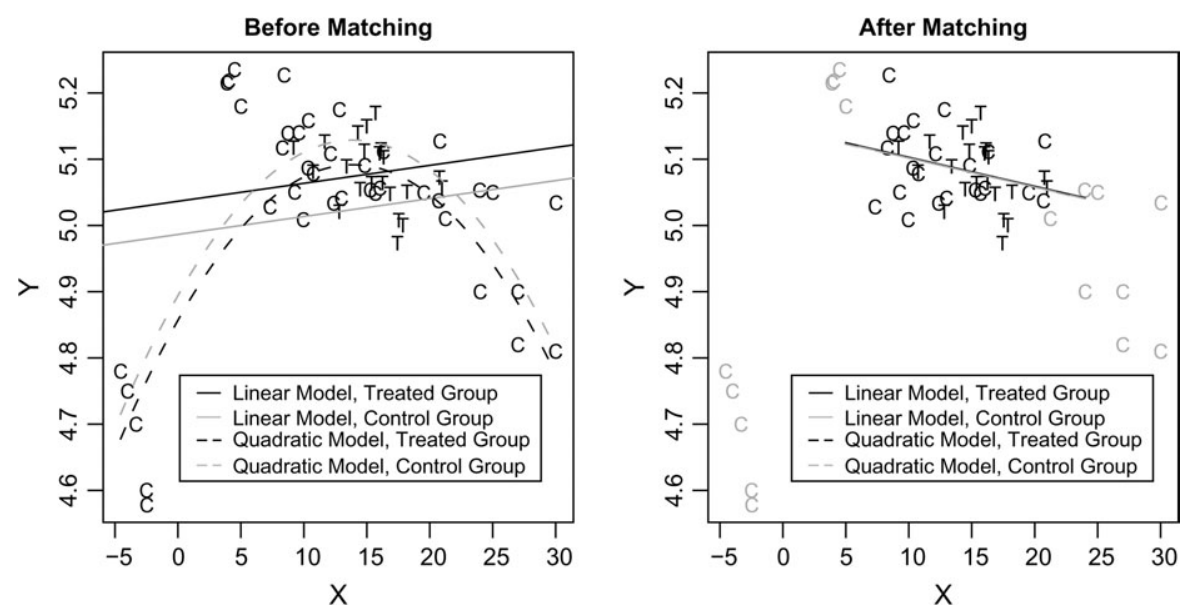

Fig. 1 Model sensitivity of ATE estimates for imbalanced raw and balanced matched data. This figure presents an artificial data set of treated units represented by " $T$ " and control units represented by "C." The vertical axis plots $Y_{i}$ and the horizontal axis plots $X_{i}$. The panels depict estimates of the ATE for a linear and quadratic specification, represented by the difference between parallel lines and parabolas, respectively. Dark lines are fitted to the treated points and gray to the controls. In the raw data, plotted in the left panel, some of the control units are far outside the range of the treated units, and these outlying control units are influential in the parametric models. In the matched data, plotted in the right panel, treated units are matched with control units that are close in $X_{i}$ (gray units are discarded), and as a result treatment effect estimates are similar regardless of model specification. The two linear and two quadratic lines also appear on the right graph (on top of one another), truncated to the location of the matched data.

\subsection{Model Dependence in Observational Data}

We first illustrate the problem of sensitivity to model specification and then give a more formal definition of model dependence. The left graph of Figure 1 plots artificial data for outcome $Y_{i}$ on the vertical axis and a pretreatment covariate $X_{i}$ on the horizontal axis (we discuss the right graph in Section 5.2). This data set was designed to illustrate the problem; in real examples, aspects of the problem we portray here often appear, but they may be more difficult to see given the simultaneous presence of other methodological problems. In addition, although a good data analyst could easily identify outliers in this onedimensional case, doing so is harder in the usual situation with many covariates. In this figure, each data point is plotted as a " $\mathrm{T}$ " for treated units $\left(T_{i}=1\right)$ and "C" for control units $\left(T_{i}=0\right)$. We then fit two regressions to these data. The first is a linear regression of $Y_{i}$ on a constant, $T_{i}$, and $X_{i}: E\left[Y_{i} \mid T_{i}, X_{i}\right]=\alpha+T_{i} \beta+X_{i} \gamma$. The fitted values for this regression are portrayed in two parallel solid lines, the dark solid line for the treated group, $E\left[Y_{i} \mid T_{i}=1, X_{i}\right]=\alpha+\beta+X_{i} \gamma$, and the gray solid line for the controls, $E\left[Y_{i} \mid T_{i}=0, X_{i}\right]=$ $\alpha+X_{i} \gamma$. The positive vertical distance between the two straight lines is this parametric model's causal effect estimate.

Model dependence is easy to see by also fitting a quadratic model to the same data, which merely involves adding an $X_{i}^{2}$ term to the original linear regression. Fitted values for the quadratic regression appear as dashed curves in the same left graph, again gray for the controls and solid black for the treated. Clearly, these fit the same data markedly differently from the original regression. Not only is the overall shape completely different, but 
the causal effect has now switched signs, which can be seen because the gray solid line is below the dark solid line, whereas the gray dashed curve is above the dark dashed curve.

Ultimately, these two models estimate the causal effect by the average vertical distance between the C's and T's. They differ only in how they compute this average. In this case, the linear model estimates a causal effect of 0.05 , whereas the quadratic model estimates a causal effect of -0.04 , and of course other models would yield other estimates. A key problem that generates this model dependence is the presence of control units far outside the range of the treated units. The model estimation thus extrapolates over a range of data that do not include treated and control units and so is particularly sensitive to the set of control units that do not look similar to the treated units. These extrapolations make causal effect estimates exquisitely sensitive to minor modifications in the statistical model (King and Zeng 2007).

Some researchers surely respond to this diversity of possible models by inadvertently choosing specifications that support their favored hypotheses. Current best practice is to portray forthrightly at least some aspects of specification uncertainty in published work by giving results for multiple specifications and evaluating how model dependent the substantive results are. But researchers of course do not often go very far in portraying the sensitivity of their causal inferences to model specification, and conveying all the sensitivity is essentially impossible.

In attempting to develop methods that indicate the degree of model dependence that could possibly occur given only a set of explanatory variables, King and Zeng (2006) "define model dependence at point $x$ as the difference, or distance, between the predicted outcome values from any two plausible alternative models ... . By 'plausible' alternative models, we mean models that fit the data reasonably well and, in particular, they fit about equally well around either the 'center' of the data (such as a multivariate mean or median) or the center of a sufficiently large cluster of data nearest the counterfactual $x$ of interest." In our case, the predicted outcome values are predicted potential outcomes, $Y_{i}(0)$ and $Y_{i}(1)$, which in our framework can be modeled separately at the parametric stage and each of which may have issues with model dependence. In practice, of course, additional model dependence will occur when models are chosen that do not fit the data. The left graph in Figure 1 obviously combines both sources of model dependence.

\section{Nonparametric Preprocessing}

The goal of matching in general and our specific nonparametric preprocessing approach in particular is to adjust the data prior to the parametric analysis so that (1) the relationship between $T_{i}$ and $X_{i}$ is eliminated or reduced and (2) little bias and inefficiency is induced. If we are able to adjust the data so that $T_{i}$ and $X_{i}$ are completely unrelated (which makes the control and treatment groups identical with respect to $X_{i}$ ), we will have moved a good deal of the way from Section 4.2 to Section 4.1. An assumption of ignorability is still necessary, but we would no longer need to model the full parametric relationship between the dependent variable and the multidimensional $X_{i}$. This also eliminates an important source of model dependence in the resulting parametric analysis stemming from the functional form specification and the curse of dimensionality. For data sets where preprocessing reduces the extent of the relationship between $T_{i}$ and $X_{i}$, but is unable to make them completely unrelated, model dependence is not eliminated but will normally be greatly reduced. If nonparametric preprocessing results in no reduction of model dependence, then it is likely that the data have little information to support causal inferences by any method, which of course would also be useful information. 


\subsection{The Immediate Goal of Preprocessing}

How can we adjust the data without inducing bias in our causal estimates? The key to this problem is that the fundamental rule for avoiding selection bias-not selecting on the dependent variable - does not prevent us from selecting observations on the explanatory variables $\left(T_{i}\right.$ or $\left.X_{i}\right)$. (Random or other physical assignments that depend on observed covariates, such as matched pair or randomized block designs, in experiments and stratified sampling in surveys are other examples of valid data collection mechanisms that select observations given chosen values of the explanatory variables.) We can also select, duplicate, or selectively drop observations from an existing sample without bias, as long as we do so using a rule that is a function only of $T_{i}$ and $X_{i}$. Our preprocessed data set will therefore include a selected subset of the observed sample for which $T_{i}$ and $X_{i}$ are unrelated, meaning that the treatment and control groups have the same background characteristics, or in other words that this relationship holds

$$
\tilde{p}(X \mid T=1)=\tilde{p}(X \mid T=0),
$$

where $\tilde{p}(\cdot)$ refers to the observed empirical density of the data, rather than a population density. ${ }^{7}$ The simplest way to understand how we can satisfy equation (11) by preprocessing is via one-to-one exact matching. The idea is to match each treated unit with one control unit for which all the values of $X_{i}$ are identical. Our preprocessed data set thus is the same as the original data set with any unmatched control units discarded and thus with $T_{i}$ and $X_{i}$ now independent. If all treated units are matched, this procedure eliminates all dependence on the functional form in the parametric analysis. (If some treated units cannot be matched, then they either need to be adjusted during parametric modeling, which of course risks extrapolation bias, or dropped, which can change the quantity of interest.) It is also highly intuitive since it directly parallels an experiment where we find pairs of units that are identical in all observable ways and assign one from each pair to be treated and the other to be a control. Then no matter what effect $X_{i}$ has on $Y_{i}$, we can ignore it entirely since $X_{i}$ is literally held constant within each pair of units.

Although one-to-one exact matching can eliminate model dependence and any bias from incorrect assumptions made during the parametric stage of analysis, it is not the only way to break the link between $T_{i}$ and $X_{i}$, since satisfying equation (11) only requires the distributions to be equivalent. Thus, to be clear, matching does not require pairing $o b$ servations (indeed, there might have been less confusion if the technique had been called "pruning"); only the distributions need be matched as closely as possible. Moreover, exact matching has the disadvantage in many applications of using relatively little of the data. Finding matches is often most severe if $X_{i}$ is high dimensional (another effect of the curse of dimensionality) or contains continuous variables. The result may then be a preprocessed data set with very few observations that leads to a parametric analysis with large standard errors. If this occurs, common practice is to use some form of inexact matching that balances as well as possible, which thus effectively sacrifices some bias reduction for the increased efficiency that comes from having more observations in the preprocessed data set. In our approach, if we lose some opportunity for bias reduction, we do so only in the preprocessing stage; the researcher's second-stage parametric analysis still has a chance to eliminate the remaining bias. Details about how to match when exact

\footnotetext{
${ }^{7}$ To be more specific, the empirical density is defined as $\tilde{p}(x)=\#\left\{i \in\{1,2, \ldots, n\}: X_{i}=x\right\} / n$, for all $x$, where $\# A$ is the number of elements in the set $A$. The corresponding denominator $n$ is $\sum_{i=1}^{n} T_{i}$ on the left side and $\sum_{i=1}^{n}\left(1-T_{i}\right)$ on the right of equation (11).
} 
one-to-one matching is infeasible appear in our software documentation; common misunderstandings of the theoretical literature on approximate matching appear in Section 6.

A key point about matching as nonparametric preprocessing is that matching is not a method of estimation: obtaining causal effect estimates from matching requires that it be paired with some analysis method. In the vast majority of applications, the analysis method has been a simple difference in means between the treatment and control groups. This method certainly makes sense in the case of exact one-to-one matching since most parametric procedures applied to exactly matched data would give the same estimates. However, with matching that is not exact, using the difference in means estimator is equivalent to assuming that any remaining imbalance in the matched sample is strictly unrelated to the treatment, which we know is false, or has no effect on the outcome, which we have no evidence about before consulting the outcome variable, and we will often have good evidence to the contrary in real analyses. Thus, we recommend that scholars make use of their decades of experience with parametric models to adjust (i.e., to interpolate or slightly extrapolate) the matched sample. The adjustment necessary is far less onerous, model dependent, and thus much more empirical than what would be necessary without matching.

\subsection{Illustration}

We now offer an example, in the right graph in Figure 1, of the reduction in model dependence produced by matching that is not exact. The data in this panel are the same as that for which the parametric analysis in the left graph gives highly model-dependent results. This is merely an illustration, easy to see in this one-dimensional case, of patterns that would be difficult to detect without matching in data sets with many variables and other methodological issues.

The difference is that the matching procedure deleted the observations that would require substantial extrapolation (marked as gray C's) and produce the imbalance. With these deletions, the data set is now highly balanced, and as such the linear model and the quadratic model give essentially identical causal effects. In the matched data, both parametric models yield estimates of approximately 0.001 (which is close to the true effect of 0 we used to generate the data). Preprocessing has therefore made the functional form assumption about whether to include $X_{i}^{2}$ in the regression largely irrelevant. Indeed, for a large range of models, this preprocessed data set will be mostly insensitive to the choice of functional form assumptions and so will return highly similar causal effect estimates. (We truncated the lines in the right graph to emphasize that we avoid extrapolation to draw causal inferences here and also limit our inferences to data in this region.)

\subsection{The Ultimate Goal of Preprocessing}

The ultimate goal of preprocessing is to help obtain accurate causal effect estimates, such as with small bias and variance. The standard approach to estimation in the social sciences presently involves parametric modeling of raw data, where a single correct model must be chosen but multiple candidate models are usually available, and so a unique estimator is not even specified ex ante and thus not well defined. Compounding the problem of defining formal properties for estimators like these in practice is that the specific choice of a model, and thus estimator, is picked by the analyst as a function of almost anything the analyst wishes, including the dependent variable or the causal effect estimates themselves. Moreover, this function is not stated.

Thus, except in unusual situations where the correct model is known, the standard parametric-only approach to causal effect estimation cannot be described as having any 
formal statistical properties other than some degree of model dependence (although in some situations, it may be possible to characterize quantities such as the maximum degree of bias). To get to the point where we can consider known properties of estimators, and ultimately produce estimates with low bias and variance, we first need to reduce model dependence.

We know from the results in Section 5.1 that matching which achieves good balance will reduce or eliminate model dependence. We also know from theoretical and simulation results that, in a wide range of scenarios, using matched samples can result in substantial bias and variance reduction, compared with using random samples of the same size (Rubin and Thomas 1992, 1996). Similarly, Imai and van Dyk (2004) found reductions in both bias and variance when using subclassification on estimated propensity scores, compared with analyses based on the full data.

To be more specific, the basic setting for which the theoretical results hold is that of affinely invariant matching methods (such as matching using the propensity score) with ellipsoidally symmetric covariate distributions (such as the normal or $t$ distribution). ${ }^{8}$ The key result is that matching in this setting is "equal percent bias reducing," meaning that it will reduce bias in all dimensions of $X$ by the same amount. Matching in this setting thus reduces bias in any function of the $X$ 's, including the outcome of interest (Rubin and Thomas 1992). The findings also lead to approximations for the reduction in the bias and variance that is possible when using matching with normally distributed covariates and a linear relationship between the covariates and the outcome, including results showing that matching on estimated propensity scores can result in additional variance reductions, relative to using the true propensity score (Rubin and Thomas 1996). The amount of bias and variance reduction possible depends on the covariate distributions in the treatment and control groups, the size of the initial bias in $X$, the original sample sizes, the number of matches selected, and the correlation between $X$ and the outcome.

We now offer an intuitive explanation of the paradoxical advantages of discarding data for reducing variance, in addition to bias. Consider first a simple linear regression with one treatment variable, $T_{i}$, and one covariate, $X_{i}$. The variance of the coefficient on the treatment variable (i.e., of the causal effect) is equal to $\sigma^{2} /\left[n(1-\gamma) s_{T}^{2}\right]$ where $\sigma^{2}$ is the conditional variance of the dependent variable, $n$ is the number of observations, $s_{T}^{2}$ is the sample variance of the treatment variable, and $\gamma$ is the coefficient from regressing $X_{i}$ on $T_{i}$. If matching improves balance, then the dependence between $T_{i}$ and $X_{i}$ will drop, $\gamma$ will be smaller than in the original sample, and hence the variance will be smaller. An analogous situation applies to nonlinear data generation processes.

The only issue is that this advantage in reducing variance by reducing $\gamma$ can be overcome by dropping $n$ too much in the process. In most situations, a little judgment and careful analysis can easily avoid the problem. We explain in four ways. First, in applications with many more control than treated units, the variance of the causal effect is mostly a function of the number of treated units, and so losing control units until their number approximates the number of treated units will not reduce the variance much, while still reducing bias. Second, for applications with roughly the same number of treatment and control units, reductions in $\gamma$ by much more than $50 \%$ due to matching are not uncommon, whereas proper matching in this situation would not normally lose anywhere near as large

\footnotetext{
${ }^{8}$ Simulation results indicate that the results hold much more generally (Rubin and Thomas 1996), and the theoretical results also hold in more general settings, including conditionally ellipsoidally symmetric distributions (such as general location models for continuous covariates with distributions like the normal or $t$ within classes defined by categorical covariates; Rubin and Thomas 1996), as well as "discriminant mixtures of proportional ellipsoidally symmetric distributions" (Rubin and Stuart 2006).
} 
a fraction of observations, and so variance usually does drop following properly applied matching.

Third, the ultimate goal of any causal estimation procedure is to reduce something like mean squared error, which is squared bias plus variance (although in observational studies bias is normally more of a concern than variance because, unlike in an experimental setting, unbiasedness is not guaranteed). Thus, even if $n$ is reduced so much that the variance increases (even as $\gamma$ decreases), matching will still be advantageous in mean squared error unless we drop $n$ to an even lower level so that it outweighs not only the reduction in variance that would otherwise occur but also the squared bias reduction. In simulations, the reduction in $n$ required to overcome the advantages in mean squared error is usually quite substantial (Rubin and Thomas 1996; Imai and van Dyk 2004). The result is that when applied carefully so that $n$ is not much smaller in the matched sample than the original sample, matching will generally reduce both bias and variance of estimates from subsequent parametric analyses.

Finally we note that, although matching discards data, it can actually increase the efficiency of estimates (Smith 1997). This may seem counterintuitive, as it would seem to violate a first principle of statistics, informally described as "more data are better." However, more data are in fact better only when using an estimator that is "self-efficient" (Meng and Romero 2003), which roughly speaking is an estimator which is based on a model that is correct (or sufficiently correct to make use of more data to improve inferences). Estimators that are not self-efficient can have variance reductions when discarding data. For a simple example, consider data generated from a univariate heteroskedastic linear-normal regression model, $Y_{i} \sim N\left(\beta_{0}+\eta T_{i}+\beta_{1} X_{i}, \sigma_{i}^{2}\right)$, for $i=1, \ldots, 1000$, and variance $\sigma_{i}^{2}$ equaling $\theta$ for $X_{i} \leq 5$ and $500 \theta$ for $X_{i}>5$, where $\theta>0$ and say 50 observations reside at $X_{i}>5$. With data generated from this model, weighted least squares is a self-efficient estimator, and thus dropping data would increase standard errors. In contrast, the least squares estimator would not be self-efficient, which can be seen because dropping the 50 observations with 500 times the variance would greatly reduce heterogeneity and thus reduce estimated standard errors. If we know the correct model, this is not an issue, but if as is usually the case we do not know the correct model and have a range of potential parametric models we might consider, self-efficiency cannot be assumed, and so dropping data can be beneficial. ${ }^{9}$

An advantage of our two-step procedure is that it is doubly robust in the sense that under weak conditions (such as ruling out extreme cases where matching leads to nonidentification even though the correct parametric model is specified), if either the matching or the parametric model is correct, but not necessarily both, causal estimates will still be consistent (see Robins and Rotnitzky 2001). That is, if the parametric model is misspecified, but the matching is correct, or if the matching is inadequate but the parametric model is correctly specified, then estimates will still be consistent. The common procedure of matching followed by an unadjusted difference in means does not possess this double robustness property.

\subsection{Summary}

The immediate goal of matching is to improve balance, the degree to which the treatment and control covariate distributions resemble each other as in equation (11), without losing

\footnotetext{
${ }^{9}$ Although we introduce this simple example to illustrate circumstances where discarding data can be beneficial, we can also use this example to illustrate another benefit of matching. In general, good balance makes $X$ irrelevant, and in this example least squares and weighted least squares would both give very similar estimates of the coefficient on $T$, which in this model is the estimated causal effect.
} 
too many observations in the process. The result of this process, when done appropriately, is considerably less model dependence, reduced potential for bias, less variance, and as a result lower mean squared error.

The main diagnostic of success in matching is also balance, as well as the number of observations remaining after matching. Trying different matching methods is not like trying different models, some of which are right and some wrong, since balance provides a reasonably straightforward objective function to maximize and choose matching solutions. Just as we iteratively evaluate a likelihood function to its optimal parameter values (and ignore any intermediate parameter values on the way to the MLEs), one should try as many matching solutions as possible and choose the one with the best balance as the final preprocessed data set. Although this point is often misunderstood (such as by occasional mistaken claims in the literature that differences across matching solutions should contribute to uncertainty estimates), matching solutions with suboptimal balance are in fact irrelevant and should play no part in our ultimate inferences. ${ }^{10}$

To ensure that selection during preprocessing depends only on $X_{i}$ (to prevent inducing bias), the outcome variable $Y_{i}$ should not be examined during the preprocessing stage. As long as $Y_{i}$ is not consulted and is not part of the rule by which one drops observations, preprocessing cannot result in stacking the deck one way or another. Experimenters typically follow a similar procedure by repeating randomization as often as desired before collecting the outcome data; if an undesirable randomization is obtained, such as with all men in the treated group and all women in the control group, they merely discard the first randomization and do it again until better balance is obtained (see Rubin 2001).

\section{Misinterpretations and Practical Implications of the Theoretical Matching Literature}

In this section, we correct instances where the theoretical literature on matching in statistics, economics, epidemiology, medicine, and biostatistics has been misunderstood by applied researchers in these and other fields. (For technical literature reviews, see Rosenbaum (2002), Imbens (2004), and Stuart (2004) and the detailed user's guide to the software that accompanies this paper described in the Appendix). We describe these issues for the ATT, so that matching is designed to choose control units that look most like the treated units.

\subsection{Selecting Covariates}

All variables in $X_{i}$ that would have been included in a parametric model without preprocessing should be included in the matching procedure. By the usual rules for avoiding omitted variable bias, these should include all variables that affect both the treatment assignment and, controlling for the treatment, the dependent variable. To avoid posttreatment bias, we should exclude variables affected by the treatment.

The theoretical literature emphasizes that including variables only weakly related to treatment assignment usually reduces bias more than it will increase variance (Rubin and Thomas 1996; Heckman et al. 1998), and so most believe that all available control variables should always be included. However, the theoretical literature has focused primarily on the case where the pool of potential control units is considerably larger than the set of

\footnotetext{
${ }^{10}$ If uncertainty remains about how to measure balance, and several solutions have almost the same balance, then one might wish to include this in our uncertainty estimates, but the decision to do so is a normative one and should be left to the investigator since all uncertainty estimation techniques exclude some sources of uncertainty.
} 
treated units. Some researchers seem to have incorrectly generalized this advice to all data sets. If, as is often the case, the pool of potential control units is not much larger than the pool of treated units, then always including all available control variables is bad advice. Instead, the familiar econometric rules apply about the trade-off between the bias of excluding relevant variables and the inefficiency of including irrelevant ones: researchers should not include every pretreatment covariate available.

\subsection{Exact Matching}

Exact matching is a powerful technique but is misunderstood in at least two ways. First, the technique is to match all control units with exactly the same covariate values. Many confuse this with one-to-one exact matching, which is unnecessarily more limited, as it uses only one control unit for each treated unit. Using all exact control matches for each treated unit reduces variance further without any increase in bias and so dominates one-toone matching. Second, some researchers only use exact matches, even if the number is very small. This procedure is flawed because it minimizes bias without regard to variance and can also lead to biased estimates of the ATT if many treated units have to be discarded because no matches are available (Rosenbaum and Rubin 1985).

If, after exact matching, a large number of units are exactly matched, then we have exact balance with little inefficiency and further matching procedures are unnecessary. Indeed, exact balance means that a difference in means is sufficient for the analysis (but to account for the difference in the number of treatment and control units, a weighted difference in means across exactly matched subclasses should be used). If an insufficient number of matches are found, we either repeat exact matching with fewer covariates or switch to other methods. In the former, we balance the included variables but do not balance at all on the rest. The excluded variables may be partially balanced due to correlations with the included variables, but some balance will be absent. In contrast, other methods, such as propensity score matching, use all variables but only approximately match.

\subsection{Common Support Problems}

Finding balance is traditionally broken into two components: ensuring common support by pruning observations where the empirical density of the control units and that for the treated units do not overlap and additional selection (or later adjustment) to make the portions of the densities that do overlap have the same heights. Areas outside of common support are particularly problematic since they require extrapolation, which can generate considerable model dependence. And indeed the farther the extrapolation is from the data, the larger model dependence can become. For example, asking in 2001 what Iraq would be like if the United States attempted to impose democracy there was pure extrapolation since the United States had not previously attempted the same thing in another country like Iraq in all relevant respects. Such an inference could only be made on the basis of theoretical modeling assumptions because relevant empirical observations from the control group did not exist.

In the applied literature, researchers often skip common support checks, which can be a major mistake. ${ }^{11}$ After all, balance can always be improved and potential model

\footnotetext{
${ }^{11}$ Some try to find common support by using the "propensity score," which we describe below. This approach may not be appropriate, however, since the propensity score can only be used to find common support when it is validated, but validation cannot occur when the data include observations outside common support (see King and Zeng 2007, and the discussion below).
} 
dependence reduced by removing units that require extrapolation. Part of the reason this step is skipped so often is that it has not until recently been clear how to identify units that require extrapolation. One conservative approach, developed by King and Zeng (2007) is to prune observations from the control group that are outside of the "convex hull" of the treatment group. With one pretreatment covariate, the convex hull of the treatment group is merely the range of the subset of observations of $X$ that are in the treatment group, so control units with $X_{i}$ greater than $\max (X \mid T=1)$ or less than $\min (X \mid T=1)$ are discarded. The general definition of the convex hull (which is more sophisticated than the range in multiple dimensions) also works to define regions of extrapolation with any number of covariates. ${ }^{12}$ Across this and the other methods of checking common support, the more conservative the approach that defines common support more restrictively, the less model dependence. Of course, more conservative approaches also leave fewer observations. For other recent ideas on identifying common support, see Iacus and Porro (2006).

\subsection{The Propensity Score Tautology}

A commonly used matching procedure is to summarize all the variables in $X$ with a single variable called the propensity score (Rosenbaum and Rubin 1983). The propensity score is the true probability of unit $i$ receiving treatment, given the covariates $X_{i}, \mathrm{e}\left(X_{i}\right)=p\left(T_{i}=\right.$ $\left.1 \mid X_{i}\right)$. It is usually estimated via a logistic regression of $T_{i}$ on a constant term and $X_{i}$ (without regard to $Y_{i}$ ). Unfortunately, the role of the propensity score in the theoretical literature differs profoundly from the way it has been widely used in practice. Understanding this disconnect, an explanation of which to our knowledge has not explicitly appeared before in the literature, is fundamental to making good practical use of this important concept.

Theoretically, the true propensity score is valuable because it is a "balancing score," meaning that if the treatment and control groups have identical propensity score distributions, then all the covariates will be balanced between the two groups. ${ }^{13}$ In addition, if treatment assignment is strongly ignorable given the covariates $X_{i}$, then it is also ignorable given only the propensity score. This means that matching can be done using just the onedimensional propensity score, instead of all the variables in $X$. Using the true propensity score in this way, as does much of the applied literature, would thus apparently solve the curse of dimensionality for matching.

In practice, however, we do not know the true propensity score (except in unusual situations like experiments). We would still be able to appeal to some of the true propensity score's theoretical properties if we had a consistent estimate of it, but such an estimate would require knowing the correct functional form for the assignment model, which is highly unlikely. Moreover, few useful theoretical results exist for the case when the true form of the propensity score equation remains unknown. These theoretical results would therefore seem to be entirely self-defeating: In order to use nonparametric matching to avoid parametric modeling assumptions, we must know the parametric functional form of the propensity score equation.

Fortunately, there is a way out. We suggest, first, looking past the theoretical properties of the propensity score, except for the purpose of motivating the goal of better propensity

\footnotetext{
${ }^{12}$ Similarly, if any treated units fall outside the convex hull of the control units, these too are often discarded. Dropping treated units changes the causal effect being estimated, and so should be done with more caution, but if it remains a relevant quantity of interest, at least it can be estimated in a reasonable way.

${ }^{13}$ The propensity score is one of many balancing scores. For example, $X$ itself is a balancing score, which explains why exact matching works.
} 
score specification, and, second and more importantly, recognizing the value of what we call the propensity score tautology. The propensity score tautology in our view is the main justification for using this technology in practice: The estimated propensity score is a balancing score when we have a consistent estimate of the true propensity score. We know we have a consistent estimate of the propensity score when matching on the propensity score balances the raw covariates. Of course, once we have balance on the covariates, we are done and do not need to look back. That is, it works when it works, and when it does not work, it does not work (and when it does not work, keep working at it).

The tautology thus provides a way to make irrelevant the knowledge of whether we have satisfied the conditions necessary to use the theoretical results about the true or consistently estimated score. The goal of matching is to achieve the best balance for a large number of observations, using any method of matching that is a function of $X_{i}$, so long as we do not consult $Y_{i}$. As it turns out, and for whatever reason, one such method that researchers sometimes find useful in some applications is based on propensity scores. The reason the propensity score approach often works in practice to balance the covariates relatively quickly may be related to its as yet unproven theoretical properties, but this conjecture is irrelevant to making valid causal inferences. At least given the current state of the literature, only the propensity score tautology is useful in practice. Other theoretical results have no direct bearing on practice.

In applications, the usual practice is to estimate the propensity score by a logistic regression of $T_{i}$ on $X_{i}$. Since we are in the situation where exact matching is insufficient, a common procedure is to match each treated unit to the control unit with the most similar value of the estimated propensity score $\hat{e}\left(X_{i}\right)$ (which is known as nearest neighbor matching on the propensity score). ${ }^{14}$ If this procedure balances $X$ (and thus satisfies the procedures for checking balance we describe below), we use it. If not, then we respecify the logistic regression by adding interactions or squared terms and match again. If that works, then we use it. If not, we try even more elaborate specifications (such as other functional forms such as CART, neural network analyses, or others) or more sophisticated matching methods (Frölich 2004; Smith and Todd 2005).

\subsection{Deciding Which Observations to Match}

The collective wisdom of the theoretical literature recommends the following three procedures for the actual process of choosing matched data sets. Unfortunately, most matching applications merely use software defaults and miss the advantages of these more sophisticated techniques.

First, if many more control than treatment units are available, choosing more than one control match for each treated unit will increase the efficiency of the procedure (although each match past the first usually reduces the variance less than the previous one) and can in some instances greatly reduce the bias too (Smith 1997). If, instead, fewer controls are available than those treated, then matching with replacement-allowing each control unit to be matched to more than one treated unit-is a good option (Dehejia and Wahba 1999). Alternatively, we can consider switching the definition of treatment and control groups

\footnotetext{
${ }^{14}$ When matching without replacement, two different approaches of matching nearest neighbors are available. The first, known as "greedy" matching, starts with some treated unit and matches the closest control unit that has not yet been matched. This approach, although slightly faster and easier to understand, is not invariant to the order in which units are matched. A second approach, known as "optimal" matching, avoids this issue by minimizing the total distance within matched units (e.g., Rosenbaum 1989). Our software implements both, incorporating optimal matching code provided by Hansen (2005).
} 
(although, if using ATT, this will change the substantive definition of the causal effect unless one uses more sophisticated estimators; Lechner 2000).

Second, we are sometimes in the situation of suspecting from prior evidence (but not from the present data set) that some covariates have a disproportionately large effect on our outcome variable. When this is the case, even slightly mismatching on these variables may severely bias our causal effect. To avoid this problem, we suggest matching using two separate metrics, one for the large-effect variables and another for the rest. If feasible, we create pools of exact matches on the large-effect variables and then use nearest neighbor matching based on the remaining variables to choose specific matches within these pools. If exact matching does not turn up sufficient observations, then we can choose the nearest neighbor on the large-effect variables, defined by the Mahalanobis distance, among all units within say 0.25 standard deviations (also known as "calipers") of the propensity score computed from all variables. ${ }^{15}$ If some of the variables in $X$ represent binary variables with very few in one category, common practice is to include them in the propensity score but not in the Mahalanobis distance calculation (Gu and Rosenbaum 1993; Rubin and Thomas 2000).

Finally, if finding a matching procedure with good balance and a large number of observations is difficult, subclassification can be a useful technique (Imai and van Dyk 2004). In subclassification, we form groups in which the distributions of covariates are the same, even though across the subclasses the distributions of covariates may be quite different. Subclassification can be accomplished by dividing the units into roughly equally sized subclasses where the estimated propensity score is, by construction, approximately constant and thus balanced. Many rely on the theoretical result that five or six subclasses are sufficient to adjust for a univariate covariate such as the propensity score (Cochran 1968; Rosenbaum and Rubin 1984), but applied researchers have not fully appreciated that as $n$ increases more subclasses are generally preferable. In addition, the number and definition of the subclasses should be tuned to the nature of the empirical distributions to ensure adequate treatment and control units in each subclass. A useful alternative is "full matching," which offers variable numbers of matches in each subclass (Hansen 2004).

\subsection{The Balance Test Fallacy}

A good matching procedure reduces bias by increasing balance, decreases the variance or at least does not increase it much, and prevents inducing new biases by matching only based on $X$ without consulting $y$ until the analysis stage. We assume that matching is based only on $X$, and checking the number of observations remaining after matching is easy. Thus, the main issue we address in this section and the next is how to evaluate balance. Conceptually, verifying balance involves checking whether equation (11), $\tilde{p}\left(X_{i} \mid T_{i}=1\right)=\tilde{p}\left(X_{i} \mid T_{i}=0\right)$, holds. One way to think about this process is to imagine, for all the variables in $X_{i}$, forming a multidimensional histogram for all the treated units and comparing it to another multidimensional histogram of all the control units. Because of the curse of dimensionality, multidimensional histograms with more than a few covariates tend to be very coarse or have many empty bins and so are difficult to evaluate and compare. Thus, researchers usually examine various low-dimensional summaries instead. If a low-dimensional summary differs between the treated and control groups, then we know equation (11) does not hold. The risk of course is that even if the treatment and control groups match according to some low-dimensional summaries, we still cannot be

\footnotetext{
${ }^{15}$ The 0.25 standard deviation figure, although not a universal constant of nature, is the most common recommendation in the literature; it appears to have been interpolated from the results in Cochran and Rubin (1973).
} 
certain that equation (11) holds since it is a multivariate concept, and so using several different checks is always a good idea.

Here we describe what Imai, King, and Stuart (2006) call the balance test fallacy, which unfortunately afflicts numerous applications of matching in most fields. The critical misunderstood point is that balance is a characteristic of the observed sample, not some hypothetical population. The idea that hypothesis tests are useful for checking balance is therefore incorrect, and $t$ statistics below 2 and $p$ values above 0.05 have no special relevance for assessing balance. But in addition, the fallacy has several serious implications. First, balance tests do not provide levels below which imbalance can be ignored: The closer the two observed treatment and control groups in the sample, the better. The problem that has been ignored is that if imbalance, no matter how small, occurs for a variable that happens to have a large enough effect on $Y$, then this tiny or "insignificant" imbalance can translate into a large bias and/or inefficiency in our causal estimates, so there is no reason to stop if you can find better balance.

More serious is that balance tests can also be highly misleading, even when using them as objective functions to optimize. In particular, pruning too many observations reduces the statistical power of a hypothesis test (i.e., the probability of rejecting the null hypothesis) and thus affects the test, even if this pruning does not improve balance at all. Imai, King, and Stuart (2006) illustrate this danger by creating a sequence of matched data sets by randomly pruning increasing numbers of control group observations. Random matching has no systematic effect on balance, but the test statistic indicates that the more data you randomly discard, the better balance gets, which is a fatal flaw. In fact, since hypothesis tests are driven in part by factors other than balance (including the number of remaining observations, the ratio of remaining treated to control units, and the variance of the treated and control groups), they are not even monotonic functions of balance: the $t$ test can get apparently better while balance gets worse, or vice versa.

\subsection{Better Matching Evaluations}

Instead of using hypothesis tests for assessing balance, we need to assess the difference in the multivariate empirical densities of $X$ for the treatment and control groups. Since working with multivariate densities is difficult, we follow the common procedure of working with lower dimensional summaries, but we do so by directly assessing differences. We also recommend that the measures applied be presented in the units of the original variables, so that the substance of the problem is emphasized and the relationship between the size of the remaining imbalance can be compared to one's views about the potential importance of the variable in question.

One particularly simple low-dimensional summary compares the mean of each variable in $X$ for the treated group with the mean of each variable in the control group. The smaller these differences are the better. One rule of thumb that has been offered is if one or more of these differ by more than a quarter of a standard deviation of the respective $X$ variable, then better balance is needed (Cochran 1968), but finding "small" imbalance in the original units is the real goal. It is also useful to compare the standard deviations of each variable between the two groups, as well as interactions or higher order moments. Another useful procedure is to compare treatment and control histograms one variable at a time or in pairs if enough data are available.

Our preferred approach is to use an empirical quantile-quantile plot, or QQ plot, for each variable (and often their interactions) to compare the full empirical distributions for the treated and control groups for each variable. QQ plots are usually the best way to 
compare two univariate distributions: They plot the quantiles of a variable of the treatment group against that of the control group in a square plot (we give an example in Section 7). We also numerically summarize these plots with mean and maximum deviation between the two distributions on the scale of the variables being measured (which is the average or maximum deviation from the 45 -degree line). ${ }^{16}$ (If one wishes to compare the balance across different covariate dimensions, then differences in empirical cumulative distribution functions can be used, instead.)

A paradoxical but sometimes useful procedure is to examine the QQ plot of the propensity scores of the control and treated units. This is paradoxical (and part of the propensity score tautology) because it relies on the propensity score as a summary of the data to check whether propensity score matching is adequate. It is useful nonetheless as one of our procedures for checking balance because it offers a low-dimensional summary not obviously worse than examining the variables one at a time. Indeed, for the reasons discussed above, it is often a good low-dimensional summary.

The immediate goal of matching is balance, which involves adjusting the data set to reduce dependence between $T_{i}$ and $X_{i}$. Since for feasibility scholars will use onedimensional summaries to substitute for the comparison of multidimensional histograms, evaluating balance should always be done in multiple ways. Relying on any one measure to assess balance will never be adequate (unless matching is exact). In addition, the ultimate goal of matching is not merely balance but reducing bias and model dependence in estimating the causal effect of $T_{i}$ on $Y_{i}$. We exclude any information from $Y_{i}$ in the matching procedure so as to avoid selection bias, but the key is that causal estimation bias and model dependence is a function of both imbalance in our covariates and our best prior information about the importance of each covariate (the effect of $X_{i}$ on $Y_{i}$ controlling for $T_{i}$ ). Obtaining good balance on covariates that are likely to be important is more crucial than those that have less of an effect since important covariates will inflate any remaining imbalance to produce more model dependence. Thus, obtaining a good matched data set requires careful assessment and evaluation through multiple objective functions (none of which involve balance hypothesis tests), as well as the combination of the quantitative measures discussed above and available prior information about the likely relative importance of each of one's covariates. Automated searches can also be useful in this regard, such as the promising approach of Diamond and Sekhon (2005), whose software makes it possible to choose appropriate balance measures (and is also incorporated in MatchIt).

If some covariates are omitted from some of the matching procedures, the balance on them should still be checked. Doing so is often a good way to discover that some covariates are indeed important, since researchers frequently have good qualitative knowledge of variables not coded in $X_{i}$, especially in nonsurvey data on countries or regions and can check balance qualitatively even when quantitative measures are not available to match on (Rosenbaum 2002, chap. 3). Indeed, preprocessing can help researchers better understand their data when supplemented by good qualitative information and research (e.g., Rosenbaum and Silber 2001).

If meeting these criteria for balance proves impossible, we then need to recognize that preprocessing by matching may not be helpful. Unfortunately, if preprocessing is

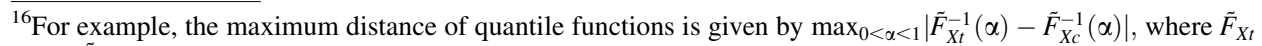
and $\tilde{F}_{X c}$ are the empirical cumulative probability distribution functions of a single variable $X$ for the matched treatment and matched control groups, respectively. That is, $\tilde{F}_{X t}(x)=\frac{1}{n^{*}} \sum_{i=1}^{n^{*}} I_{\left\{X_{i} \leq x\right\}} T_{i}$ and $\tilde{F}_{X c}(x)=$ $\frac{1}{n_{c}^{*}} \sum_{i=1}^{n^{*}} I_{\left\{X_{i} \leq x\right\}}\left(1-T_{i}\right)$, where $n_{t}^{*}=\sum_{i=1}^{n} T_{i}$ and $n_{c}^{*}=\sum_{i=1}^{n^{*}}\left(1-T_{i}\right)$, are the ${ }^{n_{t}^{*}} \sum_{i z e}$ of matched treatment and matched control groups, respectively, $n^{*}=n_{t}^{*}+n_{c}^{*}$ is the size of matched data, and $\mathrm{I}_{\{\cdot\}}$ denotes the indicator function.
} 
unhelpful, then any parametric procedure will likely require severe extrapolation and hence will be highly model dependent. In the unusual situation where particular parametric assumptions are somehow justified and verified, then it may be reasonable to proceed. In most applications, however, model sensitivity that cannot be improved by preprocessing because balance is too hard to achieve marks a data set that is too fragile for making robust causal inferences by any means.

\subsection{Parametric Outcome Analysis}

After choosing the final matched sample, preferably with maximum balance, reduced heterogeneity, and a large number of observations remaining, the parametric analysis can then proceed. Unfortunately, with few exceptions the parametric analysis chosen in practice by applied researchers after matching has been a simple difference in means (or the equivalent of a regression of $Y_{i}$ on $T_{i}$ without any control variables). This is unfortunate since the procedure assumes that $T_{i}$ and $X_{i}$ are unrelated. If the assumption is false, and it is false except in the rare case when exact matching is possible for all observations, then the result is the same for omitted variable bias that occurs whenever a potential confounding variable is ignored.

Thus, a better procedure is to use the same parametric analysis on the preprocessed data as would have been used to analyze the original raw data set without preprocessing. This can include the same maximization algorithms, the same software, the same model checking and fit procedures, and the same methods of computing and interpreting quantities of interest. The only reason to change these procedures is if best practices in statistical modeling were not followed, such as forgetting to focus on a single causal variable at a time, not avoiding posttreatment bias, etc. ${ }^{17}$ Using preprocessed data should reduce model dependence, and this too is worth checking: as one should even without preprocessing, we should check the sensitivity of causal effect estimates to changes in the specification.

\subsection{Computing Uncertainty Estimates}

Parsing what the theoretical literature on matching-based estimators says about proper methods of computing uncertainty estimates, such as standard errors or confidence intervals, is difficult without understanding a fundamental difference in perspectives that is rarely discussed. Neither perspective is right or wrong, and each is useful in some circumstances.

In the matching literature, some proposed nonparametric estimators-such as a difference in means with bias adjustment - are used to estimate the ATE after matching. Since the goal of nonparametric estimation is to make as few assumptions as possible, the variance estimation as well as point estimation tend to be based on complicated and sometimes application-specific procedures (e.g., Abadie and Imbens 2006a).

In contrast, our perspective (which is similar to the special cases analyzed by some statisticians; for example, Rubin and Thomas 2000) is to begin with what social scientists are now doing, which is estimating some form of parametric (usually regression type) model. We then ask how matching as nonparametric preprocessing can improve this current

\footnotetext{
${ }^{17}$ Standard parametric data analysis procedures only need to be changed when using subclassification, full matching, or matching with replacement. In the latter two, we must use weights to ensure that the parametric analysis reflects the actual observations. For subclassification, parametric analyses should be conducted separately within each subclass and the results combined by taking a weighted average (with weights based on the number of units in each subclass) or, if insufficient observations exist within each subclass, fit an overall model with fixed or random effects for the subclasses (Imai and van Dyk 2004).
} 
practice. Thus, since social scientists are already making parametric assumptions, we do not ask anyone following best practices to change their assumptions, and we do not reduce them either; we merely ask how to compute variance estimates in social scientists' current parametric models if the data are also preprocessed. The answer turns out to be simple: use the same variance estimator as one would normally do in using a parametric analysis.

We thus take advantage of a common feature of all the methods of computing uncertainty estimates associated with regression-type parametric methods: They are all conditional on the pretreatment variables $X_{i}$ (and $T_{i}$ ), which are therefore treated as fixed and exogenous. ${ }^{18}$ Since our preprocessing procedures modify the raw data only in ways that are solely a function of $X$, a reasonable method for defining uncertainty is to continue to treat $X$, and thus our entire preprocessing procedures, as fixed. The advantage of this definition is that we can easily compute standard errors and confidence intervals using the same methods researchers have been using with their parametric methods all along, but applied to the preprocessed instead of the raw data.

Thus, when estimating the ATT or ATE, we compute estimates of $\mu_{1}\left(X_{i}\right)$ and $\mu_{0}\left(X_{i}\right)$ and their uncertainty as usual from a parametric model applied to the preprocessed data. If computing conditional causal effects (either on average over all observations or just the average for the treated units), we set $\mu_{1}\left(X_{i}\right)=y_{i}$ if $T_{i}=1$ and use the parametric model to estimate $\mu_{0}\left(X_{i}\right)$ and its uncertainty, whereas if $T_{i}=0$, we set $\mu_{0}\left(X_{i}\right)=y_{i}$ and use the parametric model to estimate $\mu_{1}\left(X_{i}\right)$ and its uncertainty estimate.

\section{Empirical Illustrations}

We now offer two empirical illustrations of how preprocessing raw data via nonparametric matching can reduce model dependence. For pedagogical reasons, and to save space, we use different methods of checking balance via equation (11) in our two applications. A replication data file for these analyses is available in Ho et al. (2006).

\subsection{Democratic Senate Majorities and Food and Drug Administration Drug Approval Time}

An influential article by Carpenter (2002) tests a key hypothesis in the literature on institutional and partisan determinants of regulatory policy by examining several determinants of approval times for new drugs by the U.S. Food and Drug Administration (FDA). Here, for purposes of illustration, we focus on a portion of Carpenter's Hypothesis 1, which suggests that Democratic oversight of the FDA should lead to slower approval of new drugs (p. 495) and the specification of Model 1 of Table 2 (p. 499).

To test this hypothesis, Carpenter uses a log-normal survival model of approval times regressed on several causal variables of political oversight (median-adjusted Americans for Democratic Action (ADA) scores for House and Senate Committees as well as for House and Senate floors, Democratic majority in House and Senate, and Democratic Presidency) and 18 control variables including clinical and epidemiology factors and firm characteristics. ${ }^{19}$ The data set consists of 408 new drugs reviewed by the FDA, 262 of which were eventually approved. The remaining 146 drug applications were still pending at the

\footnotetext{
${ }^{18}$ These include methods based on using the asymptotic normal approximation to the likelihood function, direct simulation from the finite sampling distribution or posterior density, various frequentist bias corrections, robust Bayesian analysis involving classes of posteriors, and even nonparametric bootstrapping, among others.

${ }^{19}$ In the original paper, Carpenter (2002) uses a log-normal frailty model with a common (ungrouped) random effect. For computational simplicity, we drop the random effect. This has small effects on the quantities we estimate and no effect on our conclusions.
} 
time of data collection and hence are treated as right-censored observations. (Inferences from the censored observations are necessarily model dependent by design, and so this aspect of the problem is not influenced by the methods we introduce.) Approval time is measured in months passed from the submission of an application.

We focus on the causal effect of a Democratic majority in the Senate, one of the seven oversight variables. In particular, we estimate the in-sample (conditional) ATT. In the original analysis, the reported coefficient for the Democratic Senate majority variable is in the opposite direction of Carpenter's hypothesis and imprecisely estimated. Although not the central finding of the original article, for our purposes this variable is of particular interest because Carpenter $(2002,498)$ finds that " $[t]$ he coefficient estimate for this variable [Democratic Senate majority] is not significant in other regressions, and even switches sign when firm variables are added." We therefore examine whether the model sensitivity that prevented Carpenter from drawing solid conclusions about the Democratic Senate majority variable is reduced by preprocessing the data.

King and Zeng (2006) show that bias in making causal inferences can be decomposed into four terms: omitted variable bias, posttreatment bias, interpolation bias, and extrapolation bias. Before we analyze the data, we address each source of bias. We first consider the possibility of posttreatment bias. Carpenter's remaining six oversight variables are conceptually and statistically highly related and seem likely to be in part consequences of a Democratic Senate majority. For example, the change in the majority party of the Senate may well affect the median-adjusted ADA score in the Senate Committee. As such, we omit these variables to avoid posttreatment bias. (Posttreatment bias may still exist in this research design if other variables controlled for are consequences of a Democratic Senate majority. For example, if media coverage of a particular disease is affected by Democratic control, bias would be induced.) Although posttreatment bias is a critical issue in accurately estimating causal effects, it would affect parametric models with or without preprocessing and so is separate from our present goal of reducing model dependence; we do not pursue it further here.

Next, we examine extrapolation bias. As an initial cut, we examine whether the control units are in the convex hull of the treated units, using the method developed by King and Zeng (2006). None are. Of course this is a conservative test for common support, but it explains in part why Carpenter (2002) finds the results are highly model dependent. This situation makes Carpenter's analysis an especially difficult inference-and a hard case for us in trying to reduce model dependence - but it also informs our analysis. Since neither extrapolation bias nor omitted variable bias can be entirely eliminated without more data collection, we focus on reducing model dependence in interpolation bias and hence the overall bias by preprocessing the data.

To proceed, we estimate the propensity score using logistic regression with all covariates as linear predictors. We then discard 15 control units and 2 treated units that are outside of the common support of the estimated propensity score. Finally, we conduct one-to-one nearest neighbor propensity score matching (without replacement) while placing exact restrictions on the six binary variables (whether primary indication is a lethal condition, acute condition, and/or results in hospitalization; whether disease mainly affects men, women, and/or children). We choose one-to-one matching rather than one-to-many matching or full matching because our convex hull analysis indicates that most of the observations are likely to be outside of common support, and we want to make sure we keep only the most comparable units. This preprocessing procedure discards 102 units (10 treated units and 92 control units) from the original sample that would have required substantial, modeldependent extrapolations. The matched data set then consists of 306 observations. 
Table 1 summarizes how preprocessing can improve covariate balance. The table presents three balance measures after matching for each covariate and their percent improvement as compared to the original balance before matching. Matching substantially improves the balance of each covariate. Exact restrictions with six binary variables make their balance perfect, which is indicated by the fact that the values of all the balance measures are zero and their percent improvements are 100. Mean differences are considerably smaller for all but one covariate; empirical quantile measures also indicate a large improvement in balance for most variables. Preprocessing slightly increases the values of empirical quantile measures in two cases. Uniform improvement of balance for all covariates and all measures is unlikely unless exact matching is possible for all covariates. In this particular example, the lack of common support in the original data makes matching more difficult. Therefore, we adjust the remaining sample differences by fitting the parametric models to the preprocessed data. By choosing the matched set of control units that look most similar to the treated units, the treated-control comparison will take place only among units similar on the background variables and thus will not be affected as much by the model specification.

We now run the same log-normal survival analysis as Carpenter using the preprocessed data set. We use the same model as Carpenter's, with the exceptions noted above, such as the exclusion of the other six oversight variables. Since nothing changed other than the removal of observations that would have required highly model-dependent inferences and posttreatment variables that would have introduced another source of bias, we do not need any change in his analysis procedures. We compute MLEs, standard errors, and confidence intervals using the same procedures Carpenter did on the raw data. By applying this procedure to the preprocessed data, the estimated ATT is approximately -33.5 months with an estimated standard error of 7.5, indicating that a Democratic Senate majority significantly decreases the average approval time of new drugs. This result is of the same sign as Carpenter's estimate and thus continues to contradict the initially posited hypothesis.

Although data analysis would end here for those interested in the substantive research question at hand, for our methodological purposes we go a step further to study model dependence. For simplicity, we portray model dependence by the variability in maximum likelihood (point) estimates of the conditional in-sample ATT across different specifications of pretreatment covariates, although the same conclusions apply to most other quantities of interest too.

We obtain the MLE of the ATT for every possible specification in which the 18 covariates enter the model with the treatment indicator (i.e., all possible subsets of covariates from the 18). Even though we ignored interactions and nonlinearities (which are of course additional key aspects of model dependence), this amounts to $262,143\left(=\sum_{i=1}^{18}\left(\begin{array}{c}18 \\ i\end{array}\right)\right)$ survival analyses, all of which we ran on the raw data and then again on the preprocessed data. In practice, scholars may of course have substantive knowledge to narrow down these 262,143 specifications, but for our investigation we run each of these models on the raw data and on the preprocessed (matched) data. Figure 2 presents a kernel density plot (a smoothed histogram) for the two sets of results. The key result here is that estimates are far more model dependent using the raw data than using the matched data. For example, the variance across estimated point ATTs from the matched data (the solid curve) is less than one-tenth the size of that from the raw data (the dashed curve). The distribution of estimates for the matched data is also closer to the density of the normal distribution, which will happen when control variables included are having effects only due to random error. 


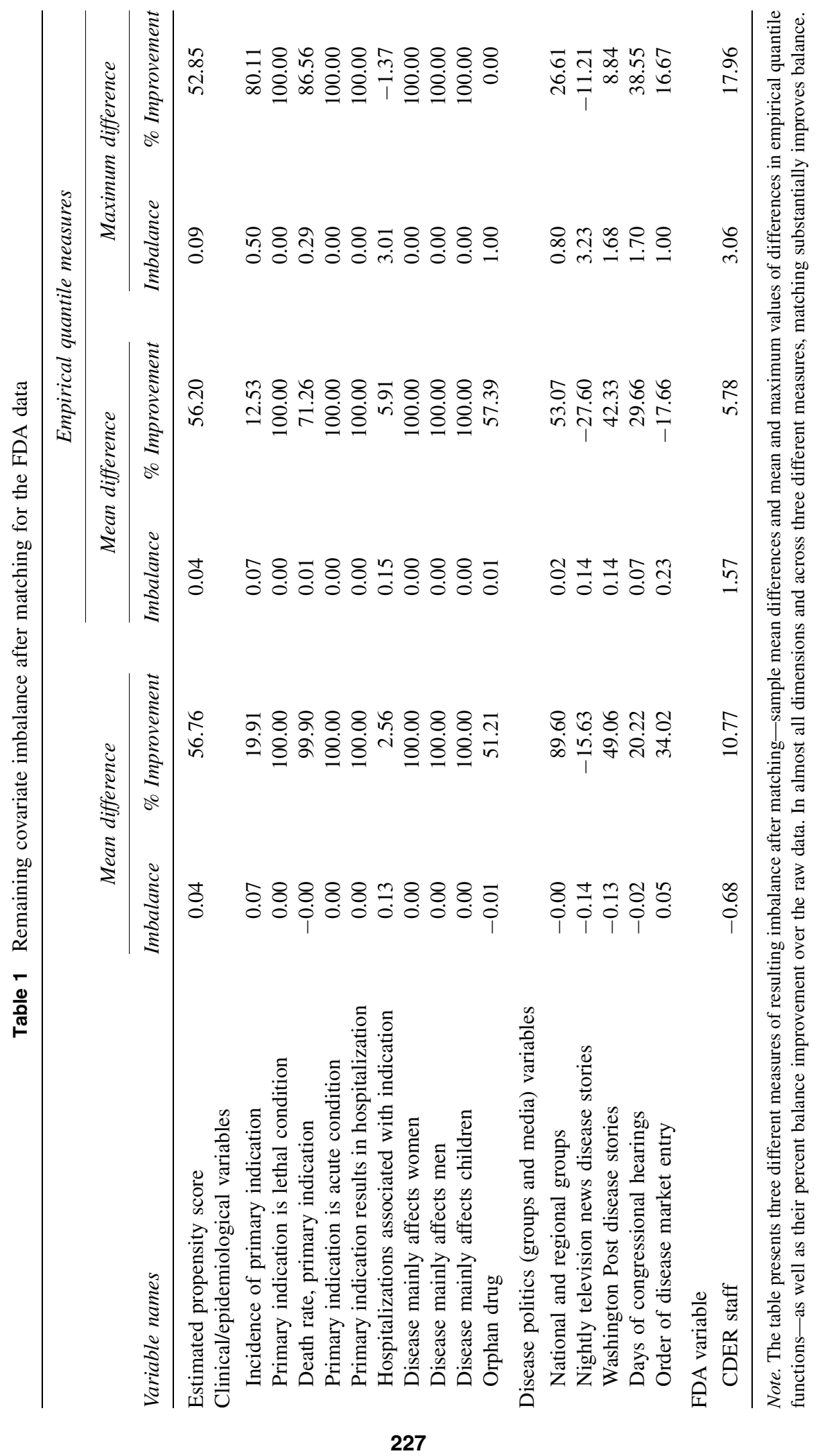




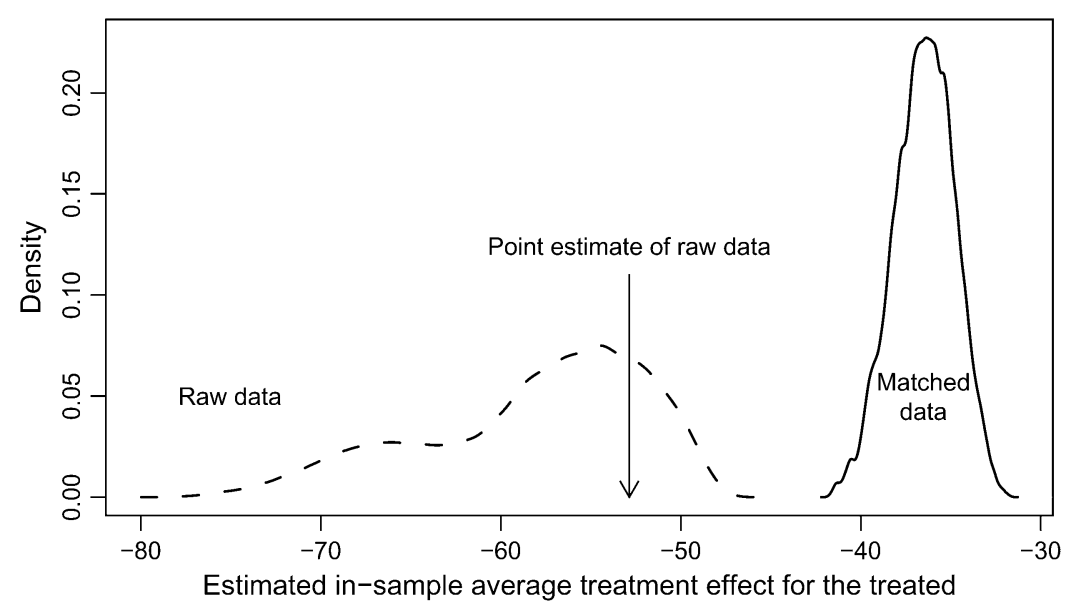

Fig. 2 Kernel density plot (a smoothed histogram) of point estimates of the in-sample ATT of the Democratic Senate majority on FDA drug approval time across 262,143 specifications. The solid line presents a density plot of the MLEs of ATT using the matched data set, whereas the dashed line is based on the raw data. The vertical arrow shows the point estimate from Carpenter's Model 1 based on the raw data. The estimate does not match Carpenter's estimate exactly because it is on a different scale and also because of the slightly different set of predictors used, as discussed above. The figure shows that ATT estimates are considerably more sensitive to model specification using the raw data as compared with the preprocessed matched data.

Figure 2 illustrates reduced sensitivity of the point estimate of the ATE. However, preprocessing also leads to a decrease in model sensitivity of the variance of the estimated treatment effect. We do not show the confidence intervals associated with each point in the density plots, however, despite the fact that matching drops more than 100 observations, preprocessing the data improves statistical efficiency. For example, the mean length of the resulting $95 \%$ confidence interval for the estimated in-sample ATT (averaged over all the 262,143 specifications) is only 43.7 for preprocessed data, which is approximately $20 \%$ shorter than the average length for the raw data. Similarly, the maximum length is 44.7 for the matched data, which is also substantially shorter than that for the raw data (63.3).

In his original analysis, Carpenter was unable to draw conclusions from the raw data due to high levels of model dependence. However, our preprocessing shows that there does exist sufficient information in the data to draw conclusions without difficult-to-justify functional form assumptions. Contrary to the original hypothesis, a Democratic Senate majority reduces the average approval time of new drugs. Using the raw data, Carpenter notes large model sensitivity, concluding that oversight covariates appear not to matter. However, the result from the matched data seems to indicate that the actual result may be relatively more firm than indicated by the usual parametric approach. One might of course still wonder why the matched estimates appear to suggest less of a difference in approval times between Democratic and Republican Senate majorities (i.e., a smaller treatment effect) than did the raw data. Two substantive explanations may be that imbalance in the raw data stemmed from the facts that the size of FDA staff and media coverage were substantially higher under Republican Senate majorities. Since staff levels and media coverage tend to decrease approval times, in the raw data more delay may have been inappropriately attributed to Democratic Senate majorities. 


\subsection{Causal Effect of Visibility on Candidate Evaluations}

For our second application, we reanalyze a study of citizen evaluations of the ideological positions of candidates for the U.S. House of Representatives by Koch (2002). The quantity of interest is the causal effect of candidate visibility on citizen voter evaluations of the candidate's ideology (scored as a seven-point ordinal scale, where high scores indicate greater conservatism). ${ }^{20} \mathrm{We}$ confine ourselves to studying the effect of visibility on Republican male candidates ${ }^{21}$ — crucial, but not identical, to the study of differences in visibility effects across gender in Koch (2002). We began by replicating the original analysis, which we did successfully.

Since randomly assigning visibility to candidates in real elections is infeasible, Koch (2002) collects observational data with pretreatment covariates including candidate ideology, voter perception of party ideology, respondent ideology, candidate feeling thermometer, and political awareness. Koch uses least squares to adjust for these covariates, making three typical assumptions, ${ }^{22}$ which, for our purposes, we do not challenge. Instead, we focus on the sensitivity of inferences to differences in specification, realizing of course that in practice qualitative evidence may help narrow the set of models. Even with a relatively small number of covariates, the curse of dimensionality looms large. Given the combinations that can be created by the unique values of these covariates and the treatment, it is easy to calculate that a parametric model that imposes no unverified functional form assumptions would require estimating 263,938,500 parameters-a problem of course in a data set with only 1203 observations. Koch reduces these parameters to only six (five main effects and one interaction term), assuming no other interactions or nonlinearities. $^{23}$

The parametric adjustments are important since more and less visible Republican male candidates differ appreciably in terms of background explanatory covariates. More visible candidates on average are more liberal than less visible candidates, who are rated 0.19 on a scale from 0 to 1 (roughly one-third of a standard deviation lower than the average rating of 0.23 of more visible candidates). In addition, more visible candidates score on average almost two-fifths of a standard deviation higher on a feeling thermometer than less visible candidates. Figure 3 gives one summary of these differences in the QQ plot of the propensity score. The QQ plot of the raw data (in black) is consistently below the 45-degree line, indicating that treated units are substantially different than control units. Model dependence is therefore likely to be a serious problem.

The original data include 853 respondents for more visible Republican male candidates, compared to only 350 respondents for less visible ones. We therefore redefine the treatment to examine the impact of a candidate being less visible on those 350 respondents.

\footnotetext{
${ }^{20}$ Visibility is measured originally by whether a candidate had campaign expenditures exceeding more than $\$ 750,000$. For simplicity and to maintain comparison to Koch (2002), we stipulate, rather than evaluate the appropriateness of, these and other measurements and methods.

${ }^{21}$ This corresponds to estimates presented in Table 2, Column 4, of Koch (2002). So as not to compare visible male candidates with less visible female candidates, we condition on gender in this illustration and focus on visibility as the key causal variable.

${ }^{22}$ First, the study assumes that visibility does not itself affect any of the pretreatment covariates. This might be violated, for example, if visibility influences the affect felt for a candidate as measured by the feeling thermometer. Controlling for the feeling thermometer would thereby induce posttreatment bias. Second, the study assumes that the visibility of one candidate does not affect the potential outcomes of another candidate. Visibility of a candidate might, for example, detract local media attention from a candidate in an adjoining district, violating independence. Third, the study assumes that "visibility" is the same treatment for all candidates.

${ }^{23}$ As originally modeled, the analysis interacts gender and visibility as the treatment of interest, but as we condition on gender the treatment becomes visibility alone.
} 


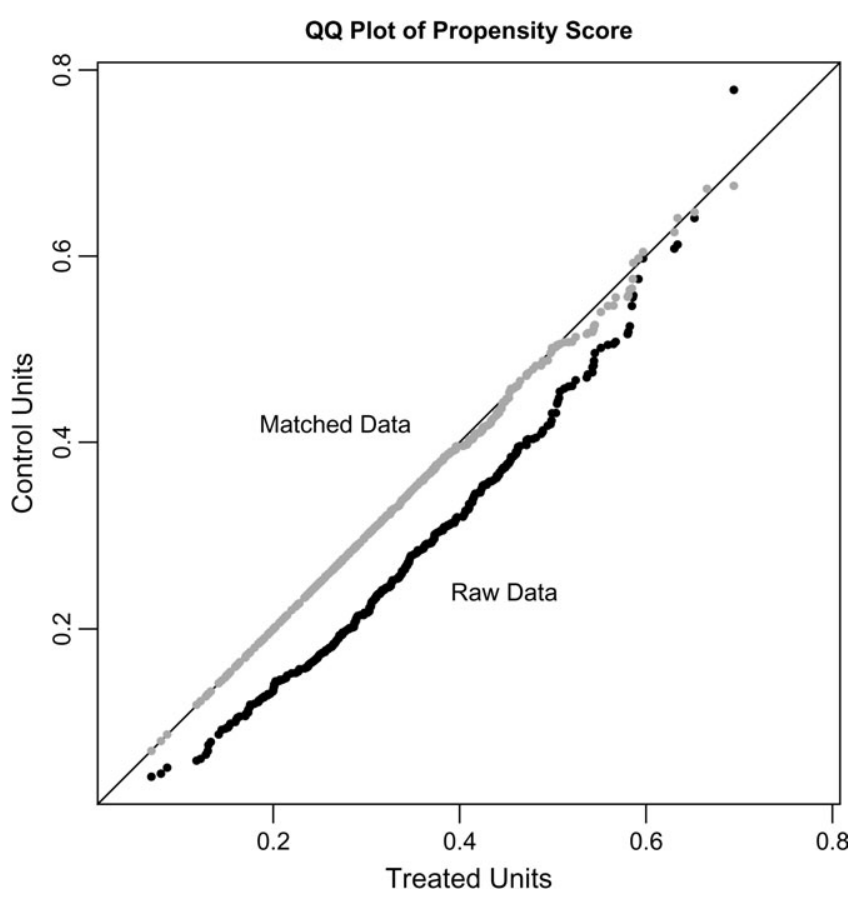

Fig. 3 QQ plot of propensity score for candidate visibility. The black dots represent empirical QQ estimates for the raw data. The gray dots represent QQ estimates for the matched data. The 45-degree line indicates identical distributions. The propensity score is estimated with a logistic regression of treatment (less visibility) on candidate ideology, voter perception of party ideology, respondent ideology, candidate feeling thermometer, and political awareness.

Through experimentation, we find that propensity score matching improves balance substantially. We estimate the propensity score via a logistic regression of visibility on all six pretreatment covariates. We conduct one-to-one nearest neighbor matching based on the estimated propensity score, resulting in 350 respondents for more visible candidates matched with 350 respondents for less visible candidates. As Figure 3 demonstrates, matching leads to treatment and control group values of the estimated propensity score being identical at almost every quantile, with gray dots lining up along the 45-degree line. The percent balance improvement in mean differences is substantial, ranging at worst from approximately $75 \%$ in perception of party ideology, political awareness, and respondent ideology, and at best to $96.0-98.3 \%$ for the remaining covariates.

With this preprocessed data set, we can now run the comparable least squares analysis as in Koch (2002). The only difference is that we run the analysis for the matched data set. This analysis yields an estimated ATE of -0.04 with an estimated standard error of 0.07 , suggesting that there is not much of an effect of less visibility on Republican male candidates.

We now turn to our main (methodological) purpose of evaluating model sensitivity by studying the variance of causal effect point estimates for $63\left(=\sum_{i=1}^{6}\left(\begin{array}{c}6 \\ i\end{array}\right)\right)$ regressions containing all possible subsets of Koch's six covariates, again for simplicity restricting ourselves to only permutations of the possible main effects. We thereby estimate 63 separate point estimates for the raw and for the preprocessed data. 


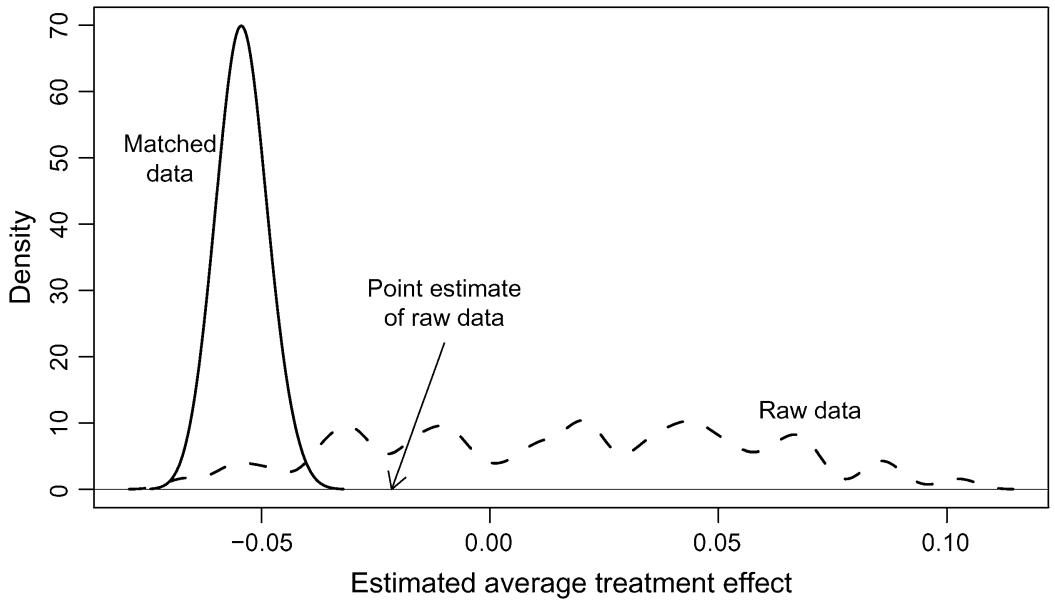

Fig. 4 Kernel density plot of point estimates of the effect of being a less visible male Republican candidate across 63 possible specifications with the Koch data. The dashed line presents estimates for the raw data set and the solid line for the matched data set. The vertical arrow presents the point estimate of the regression comparable to the one presented in the original paper. This figure shows that treatment effect estimates are much more sensitive to model specification for the raw data set compared to the matched data set.

Figure 4 plots densities of the ATTs from the raw and preprocessed data. As expected, estimates are much less variable in the preprocessed data. Estimated effects from the raw data (see the dashed line) range from -0.07 to 0.10 , signifying that low visibility for male candidates has no predictable effect on whether voters perceive candidates as more liberal. The point estimate from the raw data (or any one point estimate) cannot represent the enormous variability in these results. Estimates from the preprocessed data stand in stark contrast to the estimates from the raw data. The variability of coefficient estimates is substantially smaller, ranging from -0.06 to -0.04 , every one of which indicates that less visibility reduces voters' perceptions of the candidates as liberal. More importantly from our perspective, the standard deviation across specifications of the raw data is 10 times as large as for the preprocessed data. On average, point estimates are also lower for the matched data, suggesting that preexisting differences in feeling thermometer may have been inappropriately attributed to visibility in the raw data.

\section{What Can Go Wrong}

The advantage of matching is that it is relatively robust to small changes in procedures and produces a data set that is by design less sensitive to modeling assumptions. However, like any method, using it badly or to ill effect is certainly possible. Thus, in this section, we discuss four ways in which preprocessing can go wrong and how researchers might try to avoid these problems.

First, since the curse of dimensionality affects balancing diagnostics, we may well miss a higher dimensional aspect of imbalance when checking lower dimensional summaries. Even if we are uninterested in testing these with our parametric model, they can affect our estimates. Such will be the case with parametric models with or without preprocessing, and so in all but the most unusual cases preprocessing should at least not make things worse. One pathological case where preprocessing could hurt is if some covariate has 
a huge effect on the outcome variable and preprocessing slightly reduces balance on this variable but improves it for all the others. A researcher might be fooled into choosing a matching trade-off like this if he or she were not aware of the large effect of this covariate. Carefully evaluating what covariates are likely to have the largest effects, and using multiple measures of balance, are essential to avoid this pitfall.

Second, as with all statistical methods, a bias-variance trade-off exists for matching. If we drop many observations during preprocessing, and balance is not substantially improved, the mean squared error (or other mean-variance summary) of the estimated causal effect might actually increase. Users must pay close attention to this trade-off during the process of matching, but unfortunately no precise rules exist for how to make these choices. In particular, the methodological literature offers no formal estimates of mean squared error and so in marginal cases it can be difficult to know whether or how much preprocessing will help. Of course, dropping observations does not necessarily mean that preprocessing is worse since improving balance can also increase efficiency, and in any event including imbalanced observations requiring extrapolation in a parametric analysis merely produces false precision. So although estimated standard errors may increase in some cases with preprocessing, they would likely be more accurate. Moreover, in many situations, eliminating observations far from the rest of the data as matching does will reduce heterogeneity and thereby further reduce variance.

Third, the matching literature offers a large number of possible and seemingly ad hoc procedures. From one perspective, we might be concerned about the sensitivity of our results to changes in this process, just as we have been concerned with the sensitivity of causal effect estimates to parametric modeling assumptions. This is not normally viewed as a major issue since the right procedure is the one that maximizes balance (with $n$ as large as possible), no matter how many procedures we try. By applying this criterion in a disciplined way (i.e., without consulting $Y$ ) to a large number of possible matching procedures, no choices are open to the analyst. Instead, researchers should merely run as many as possible and choose by maximizing balance. Unlike parametric modeling exercises, we need not choose this matching procedure or another; we merely run as many as feasible, particularly those most likely to reduce bias and model dependence, and apply this criterion.

Finally, by dropping observations, we may wind up losing some critically important cases or may change either the information base of our sample or, in special cases such as when dropping treated units, the definition of the causal effect. Examining the dropped cases provides an easy diagnostic for this problem. However, we must be alert to the problem that if we learn that some critical units are dropped, then it may mean that no appropriate matches can be found for them. In this situation, we may be forced to conclude that the data do not contain sufficient information to answer the questions posed, no matter what method is chosen.

\section{Concluding Remarks}

Anyone using a parametric statistical technique for long enough (and it does not take very long) will recognize the difficulty of choosing which of hundreds of possible regressions to present in a written work. This choice is difficult, fraught with ethical and methodological dilemmas, and not covered in any serious way in classical statistics texts. Parametric methods merely assume that we know the correct specification. In practice, the "correct" specification is chosen after looking at the estimates, and so it is never clear to a reader whether an article is a true test of a hypothesis, in the sense that the author was vulnerable 
to being proved wrong, or whether the article is merely a proof of the existence of at least one specification consistent with the author's favored hypothesis. Researchers are often frustrated with how their key causal estimates depend on specification decisions they have not thought about and on which they have few real opinions.

We provide a way around at least part of this problem. Preprocessing raw data with the matching procedures we recommend makes familiar parametric methods a much more reliable tool of empirical analysis and, in particular, causal effect estimates become far more insensitive to seemingly arbitrary choices in model specification. If we read an article demonstrating that balance has been achieved for a data set, readers can worry considerably less that slightly different specifications than those discussed in the text will greatly alter its empirical conclusions. Analysts using preprocessing have two chances to get their analyses right, in that if either the matching procedure or the subsequent parametric analysis is specified correctly (and even if one of the two is incorrectly specified), causal estimates will still be consistent.

\section{Appendix: Matching Software}

A variety of excellent software is available to perform matching (Parsons 2000, 2001; Abadie et al. 2002; Becker and Ichino 2002; Bergstralh and Kosanke 2003; Leuven and Sianesi 2004; Sekhon 2004; Hansen 2005). However, each program implements only a specialized subset of available statistical procedures. Moreover, they are spread over a range of different languages and packages, which normally makes it impractical to use more than one for any applied project. Thus, as a companion to and in the same spirit as this paper, we have written software (called MatchIt, available at http://gking.harvard.edu/ matchit) that implements the vast majority of the matching procedures suggested in the diverse scholarly literatures on this subject. Where possible, MatchIt builds on and incorporates existing packages and, across all the specialized techniques, MatchIt offers a single, simple, and unified syntax. Adding procedures to MatchIt is also easy.

MatchIt operates with a single command that takes an existing data set and produces as output a single preprocessed matched data set. The preprocessed data set can then be used by standard parametric software just as one would have used the original data set. MatchIt also works seamlessly with the general-purpose statistics program, Zelig (Imai, King, and Lau 2006), so that output from MatchIt can be fed into Zelig without any extra steps. MatchIt and Zelig are freely available and run under many operating systems via the open source and free statistical program R.

\section{References}

Abadie, Alberto, David Druckker, Jane Leber Herr, and Guido W. Imbens. 2002. Implementing matching estimators for average treatment effects in stata. The Stata Journal 1:1-18.

Abadie, Alberto, and Guido Imbens. 2006a. Estimation of the conditional variance in paired experiments. KSG working paper. http://ksghome.harvard.edu/ .aabadie.academic.ksg/cve.pdf (accessed September 1, 2006).

. 2006b. Large sample properties of matching estimators for average treatment effects. Econometrica 74:235-67.

Becker, Sascha O., and Andrea Ichino. 2002. Estimation of average treatment effects based on propensity scores. The Stata Journal 2:358-77.

Bergstralh, Erik, and Jon Kosanke. 2003. dist, gmatch, and vmatch: SAS macros. Mayo Clinic, Division of Biostatistics. http://mayoresearch.mayo.edu/mayo/research/biostat/sasmacros.cfm (accessed September 1, 2006). Bishop, Christopher M. 1995. Neural networks for pattern recognition. Oxford: Oxford University Press.

Black, Dan A., and Jeffrey A. Smith. 2004. How robust is the evidence on the effects of college quality? Evidence from matching. Journal of Econometrics 121:99-124. 
Carpenter, Daniel P. 2002. Groups, the media, agency waiting costs, and FDA drug approval. American Journal of Political Science 46(July):490-505.

Cochran, William G. 1968. The effectiveness of adjustment by subclassification in removing bias in observational studies. Biometrics 24:295-313.

Cochran, William G., and Donald B. Rubin. 1973. Controlling bias in observational studies: A review. Sankhya: The Indian Journal of Statistics, Series A 35(Part 4):417-66.

Cox, David R. 1958. Planning of experiments. New York: John Wiley.

Dehejia, Rajeev H., and Sadek Wahba. 1999. Causal effects in nonexperimental studies: Re-evaluating the evaluation of training programs. Journal of the American Statistical Association 94(December):1053-62.

Diamond, Alexis, and Jasjeet Sekhon. 2005. Genetic matching for estimating causal effects: A new method of achieving balance in observational studies. http://sekhon.berkeley.edu/ (accessed September 1, 2006).

Fisher, Ronald A. 1935. The design of experiments. London: Oliver and Boyd.

Frölich, Markus. 2004. Finite sample properties of propensity score matching and weighting estimators. Review of Econometrics and Statistics 86:77-90.

Glazerman, Steve, Dan M. Levy, and David Myers. 2003. Nonexperimental versus experimental estimates of earnings impacts. The Annals of the American Academy of Political and Social Science 589(September): 63-93.

Goldberger, Arthur. 1991. A course in econometrics. Cambridge, MA: Harvard University Press.

Gu, Xing S., and Paul R. Rosenbaum. 1993. Comparison of multivariate matching methods: Structures, distances, and algorithms. Journal of Computational and Graphical Statistics 2:405-20.

Hansen, Ben B. 2004. Full matching in an observational study of coaching for the SAT. Journal of the American Statistical Association 99:609-18.

- 2005. Optmatch: Software for optimal matching. http://www.stat.lsa.umich.edu/ $\sim$ bh/optmatch.html (accessed September 1, 2006).

Heckman, James J., Hidehiko Ichimura, Jeffrey A. Smith, and Petra E. Todd. 1998. Characterizing selection bias using experimental data. Econometrica 66:1017-98.

Heckman, James J., Hidehiko Ichimura, and Petra E. Todd. 1997. Matching as an econometric evaluation estimator: Evidence from evaluating a job training program. Review of Economic Studies 64:605-54.

Heckman, James J., and Richard Robb. 1985. Alternative methods for evaluating the impacts of interventions. In Longitudinal analysis of labormarket data, ed. J. Heckman and B. Singer; Cambridge: Cambridge University Press.

Hirano, Keisuke, Guido W. Imbens, and Geert Ridder. 2003. Efficient estimation of average treatment effects using the estimated propensity score. Econometrica 71(July):1161-89.

Ho, Daniel E., Kosuke Imai, Gary King, and Elizabeth A. Stuart. 2006. Replication data set for 'matching as nonparametric preprocessing for reducing model dependence in parametric causal inference'. http:// id.thedata.org/hdl\%3A1902.1\%2FYVDZEQIYDS hdl:1902.1/YVDZEQIYDS UNF:3:QV0mYCd8eV+mJg WDnYct5g $==$ Murray Research Archive [distributed by DDI].

Hoeting, Jennifer A., David Madigan, Adrian E. Raftery, and Chris T. Volinsky. 1999. Bayesian model averaging: A tutorial. Statistical Science 14:382-417.

Holland, Paul W. 1986. Statistics and causal inference. Journal of the American Statistical Association 81:945-60.

Iacus, Stefano, and Giuseppe Porro. 2006. Random recursive partitioning: A matching method for the estimation of the average treatment effect. UNIMI-Research Papers in Economics, Business, and Statistics. Economics. Working paper 9. http://services.bepress.com/unimi/economics/art9 (accessed September 1, 2006).

Imai, Kosuke, and Gary King. 2004. Did illegal overseas absentee ballots decide the 2000 U.S. presidential election? Perspectives on Politics 2(September):537-49.

Imai, Kosuke, Gary King, and Olivia Lau. 2006. Zelig: Everyone's statistical software. http://gking.harvard.edu/ zelig (accessed September 1, 2006).

Imai, Kosuke, Gary King, and Elizabeth A. Stuart. 2006. Misunderstandings among experimentalists and observationalists: Balance test fallacies in causal inference. http://gking.harvard.edu/files/abs/matchse-abs.shtml (accessed September 1, 2006).

Imai, Kosuke, and David A. van Dyk. 2004. Causal inference with general treatment regimes: Generalizing the propensity score. Journal of the American Statistical Association 99(September):854-66.

Imbens, Guido W. 2004. Nonparametric estimation of average treatment effects under exogeneity: A review. Review of Economics and Statistics 86:4-29.

King, Gary. 1989. Unifying political methodology: The likelihood theory of statistical inference. Ann Arbor: Michigan University Press.

King, Gary, James Honaker, Anne Joseph, and Kenneth Scheve. 2001. Analyzing incomplete political science data: An alternative algorithm for multiple imputation. American Political Science Review 95(March):49-69. http://gking.harvard.edu/files/abs/evil-abs.shtml (accessed September 1, 2006). 
King, Gary, Robert O. Keohane, and Sidney Verba. 1994. Designing social inquiry: Scientific inference in qualitative research. Princeton, NJ: Princeton University Press.

King, Gary, and Langche Zeng. 2006. The dangers of extreme counterfactuals. Political Analysis 14:131-59. http://gking.harvard.edu/files/abs/counterft-abs.shtml (accessed September 1, 2006).

- 2007. When can history be our guide? The pitfalls of counterfactual inference. International Studies Quarterly (March). http://gking.harvard.edu/files/abs/counterf-abs.shtml.

Koch, Jeffrey M. 2002. Gender stereotypes and citizens' impressions of house candidates' ideological orientation. American Journal of Political Science 46:453-62.

Lechner, Michael. 2000. A note on the common support problem in applied evaluation studies. University of St. Galen. http://www.siaw.unisg.ch/lechner (accessed September 1, 2006).

Leuven, Edwin, and Barbara Sianesi. 2004. PSMATCH2: Stata module to perform full Mahalanobis and propensity score matching, common support graphing, and covariate imbalance testing. EconPapers. http:// econpapers.repec.org/software/bocbocode/S432001.htm (accessed September 1, 2006).

Lewis, David K. 1973. Counterfactuals. Cambridge, MA: Harvard University Press.

McCullagh, Peter, and James A. Nelder. 1989. Generalized linear models. 2nd ed. Monograph on statistics and applied probability 37. New York (NY): Chapman \& Hall/CRC.

Meng, Xiao-Li, and Martin Romero. 2003. Discussion: Efficiency and self-efficiency with multiple imputation inference. International Statistical Review 71:607-18.

Neyman, Jerzy. 1935. Statistical problems in agricultural experiments. Supplement to the Journal of the Royal Statistical Society 2:107-80.

Parsons, Lori S. 2000. Using SAS software to perform a case-control match on propensity score in an observational study. http://www2.sas.com/proceedings/sugi25/25/po/25p225.pdf (accessed September 1, 2006).

2001. Reducing bias in a propensity score matched-pair sample using greedy matching techniques. http://www2.sas.com/proceedings/sugi26/p214-26.pdf (accessed September 1, 2006).

Quandt, Richard. 1972. Methods of estimating switching regressions. Journal of the American Statistical Association 67:306-10.

Robins, James M., and Andrea Rotnitzky. 2001. Comment on the Peter J. Bickel and Jaimyoung Kwon, 'Inference for semiparametric models: Some questions and an answer'. Statistica Sinica 11:920-36.

- Forthcoming. Inverse probability weighting estimation in survival analysis. Encyclopedia of Biostatistics.

Rosenbaum, Paul R. 1984. The consequences of adjusting for a concomitant variable that has been affected by the treatment. Journal of the Royal Statistical Society, Series A 147:656-66.

1986. Dropping out of high school in the United States: An observational study. Journal of Educational Statistics 11:207-24.

- 1989. Optimal matching for observational studies. Journal of the American Statistical Association 84:1024-32. 2002. Observational studies. 2nd ed. New York: Springer.

Rosenbaum, Paul R., and Donald B. Rubin. 1983. The central role of the propensity score in observational studies for causal effects. Biometrika 70:41-55.

- 1984. Reducing bias in observational studies using subclassification on the propensity score. Journal of the American Statistical Association 79:516-24. 1985. The bias due to incomplete matching. Biometrics 41:103-16.

Rosenbaum, Paul R., and Jeffrey H. Silber. 2001. Matching and thick description in an observational study of mortality after surgery. Biostatistics 2:217-32.

Roy, A. D. 1951. Some thoughts on the distribution of earnings. Oxford Economic Papers 3:135-46.

Rubin, Donald B. 1973. The use of matched sampling and regression adjustment to remove bias in observational studies. Biometrics 29:185-203.

- 1974. Estimating causal effects of treatments in randomized and nonrandomized studies. Journal of Educational Psychology 6:688-701. 34-58.

1979. Using multivariate matched sampling and regression adjustment to control bias in observational studies. Journal of the American Statistical Association 74:318-28.

- 1987. Multiple imputation for nonresponse in surveys. New York: John Wiley.

2001. Using propensity scores to help design observational studies: Application to the tobacco litigation. Health Services \& Outcomes Research Methodology 2(December):169-88.

Rubin, Donald B., and Elizabeth A. Stuart. 2006. Affinely invariant matching methods with discriminant mixtures of proportional ellipsoidally symmetric distributions. Annals of Statistics 34:1814-1826. 
Rubin, Donald B., and Neal Thomas. 1992. Characterizing the effect of matching using linear propensity score methods with normal distributions. Biometrika 79:797-809.

1996. Matching using estimated propensity scores, relating theory to practice. Biometrics 52:249-64. 2000. Combining propensity score matching with additional adjustments for prognostic covariates. Journal of the American Statistical Association 95:573-85.

Sekhon, Jasjeet S. 2004. Multivariate and propensity score matching software. http://jsekhon.fas.harvard.edu/ matching/ (accessed September 1, 2006).

Smith, Herbert L. 1997. Matching with multiple controls to estimate treatment effects in observational studies. Sociological Methodology 27:325-53.

Smith, Jeffrey A., and Petra E. Todd. 2005. Does matching overcome LaLonde's critique of nonexperimental estimators? Journal of Econometrics 125(March-April):305-53.

Stuart, Elizabeth A. 2004. Matching methods for estimating causal effects using multiple control groups. Ph.D. thesis, Department of Statistics, Harvard University. 